\title{
CLASSICAL SOLUTIONS FOR THE EQUATIONS MODELLING THE MOTION OF A BALL IN A BIDIMENSIONAL INCOMPRESSIBLE PERFECT FLUID
}

\author{
Jaime H. Ortega ${ }^{1}$, Lionel Rosier ${ }^{2}$ And TAKéo TAKahashi ${ }^{2}$
}

\begin{abstract}
In this paper we investigate the motion of a rigid ball in an incompressible perfect fluid occupying $\mathbb{R}^{2}$. We prove the global in time existence and the uniqueness of the classical solution for this fluid-structure problem. The proof relies mainly on weighted estimates for the vorticity associated with the strong solution of a fluid-structure problem obtained by incorporating some dissipation.
\end{abstract}

Mathematics Subject Classification. 35Q35, 76B03, 76B99.

Received: January 8, 2004. Revised: November 23, 2004.

\section{INTRODUCTION}

We consider a rigid body occupying an open bounded set $B(t) \subset \mathbb{R}^{2}$ and which is surrounded by a perfect homogeneous fluid. We denote by $\Omega(t)=\mathbb{R}^{2} \backslash \overline{B(t)}$ the domain occupied by the fluid. In order to simplify the problem, we also assume that $B(t)$ is a disc of radius 1 and that the fluid and the solid are homogeneous. The motion of the fluid is modelled by Euler equations and the equation describing the motion of the rigid body is obtained by applying the principle of conservation of momentum. More precisely, the equations modelling the dynamics of the system read

$$
\begin{aligned}
\frac{\partial u}{\partial t}+(u \cdot \nabla) u+\nabla p=0, & \text { in } \Omega(t) \times[0, T], \\
\operatorname{div} u=0, & \text { in } \Omega(t) \times[0, T], \\
u \cdot n=h^{\prime} \cdot n, & \text { on } \partial B(t) \times[0, T], \\
m h^{\prime \prime}=\int_{\partial B(t)} p n \mathrm{~d} \Gamma, & \text { in }[0, T], \\
u(y, 0)=a(y), & \forall y \in \Omega(0), \\
h(0)=0 \in \mathbb{R}^{2}, & h^{\prime}(0)=b \in \mathbb{R}^{2} .
\end{aligned}
$$

Keywords and phrases. Euler equations, fluid-rigid body interaction, exterior domain, classical solutions.

${ }^{1}$ Universidad de Chile, Facultad de Ciencias Físicas y Matemáticas, Centro de Modelamiento Matemático, UMI 2807 CNRS-UChile, Casilla 170/3, Correo 3, Santiago, Chile and Universidad del Bío-Bío, Facultad de Ciencias, Departamento de Ciencias Básicas, Casilla 447, Campus Fernando May, Chillán, Chile. jortega@dim.uchile.cl

2 Institut Elie Cartan, Université Henri Poincaré Nancy 1, BP 239, 54506 Vandœuvre-lès-Nancy Cedex, France.

rosier@iecn.u-nancy.fr; takahash@iecn.u-nancy.fr 
In the above equations, $u$ (resp., $p$ ) is the velocity field (resp., the pressure) of the fluid, and $h$ denotes the position of the centre of the ball. Note that we have assumed that the ball is centred at the origin at $t=0$. We have denoted by $n$ the unit outward normal to $\partial \Omega$. The constant $m$ is the mass of the ball.

Let us point out that the equation for the rotation of the rigid ball has been omitted in the system above; indeed, thanks to the particular shape of the rigid body, the angular velocity of the rigid body remains constant as time proceeds.

The main difficulties when studying this problem are that the system (1.1)-(1.5) is nonlinear, strongly coupled and that the domain of the fluid is variable (and depends on $h$ ). These difficulties occur in any fluid-structure problem. In the last decade, several papers have been devoted to the existence of solutions for such systems when the fluid motion is governed by Navier-Stokes equations; to quote a few, Desjardins and Esteban $[5,6]$, Conca, San Martín and Tucsnak [2], Gunzburger, Lee and Seregin [16], Hoffmann and Starovoitov [18, 19], Grandmont and Maday [15], San Martín, Starovoitov and Tucsnak [26], Feireisl [7-9], Takahashi [30] (in the case of a bounded domain) and Serre [27], Judakov [20], Silvestre [28], Takahashi-Tucsnak [31], Galdi and Silvestre [11] (in the case where the fluid-rigid body system fills the whole space). The stationary problem was studied in Serre [27] and in Galdi [10]. Zuazua and Vázquez [33] have also tackled, in a simplified 1D model, the asymptotic behaviour of solutions.

On the other hand, as far as we know, there is no paper devoted to the existence of a (weak or classical) solution for the fluid-rigid body system in the case where the fluid is assumed to be perfect (no viscosity). However, a theory providing classical solutions to this kind of problems seems desirable for control purposes, as most of the control results for Euler flows involve classical solutions. (See e.g. Coron [3,4], and Glass [14].)

In order to write the equations of the fluid in a fixed domain, we use a change of variables. Denoting by $B$ the initial subset occupied by the solid and by $\Omega=\mathbb{R}^{2} \backslash \bar{B}$ the initial domain occupied by the fluid, we set

$$
v(y, t)=u(y+h(t), t), \quad q(y, t)=p(y+h(t), t), \quad l(t)=h^{\prime}(t) .
$$

Then, the functions $(v, q, l)$ satisfy the following system:

$$
\begin{aligned}
\frac{\partial v}{\partial t}+(v \cdot \nabla) v-(l \cdot \nabla) v+\nabla q=0, & \text { in } \Omega \times[0, T], \\
\operatorname{div} v=0, & \text { in } \Omega \times[0, T], \\
v \cdot n=l \cdot n, & \text { on } \partial B \times[0, T], \\
m l^{\prime}=\int_{\partial B} q n \mathrm{~d} \Gamma, & \text { in }[0, T], \\
v(y, 0)=a(y), & \forall y \in \Omega, \\
l(0)=b . &
\end{aligned}
$$

Let us point out that for a rigid body with an arbitrary form the additional term $r\left(v^{\perp}-y^{\perp} \cdot \nabla v\right)$ has to be incorporated in the left hand side of (1.8), where $r$ denotes the angular velocity of the rigid body. Clearly, this term is difficult to handle as $|y| \rightarrow \infty$. The existence of a classical solution for this more general problem will be investigated elsewhere.

Before stating the main result of the paper, we introduce certain notations borrowed from Kikuchi [22]. If $V$ denotes any scalar-valued function space and $u=\left(u_{1}, u_{2}\right)$ is any vector-valued function, we shall say that $u \in V$ if $u_{i} \in V$ for all $i$, for the sake of simplicity. Let $T$ be any positive number, and let $Q_{T}=\Omega \times(0, T) . \mathcal{B}(\bar{\Omega})$ (resp., $\mathcal{B}\left(\overline{Q_{T}}\right)$ ) is the Banach space of all real-valued, continuous and bounded functions defined on $\bar{\Omega}$ (resp. $\overline{Q_{T}}$ ), endowed with the $L^{\infty}$ norm. For any $\theta>0, L_{\theta}^{1}(\Omega)$ denotes the space of (class of) measurable functions $\omega$ on $\Omega$ such that

$$
\|\omega\|_{L_{\theta}^{1}(\Omega)}:=\int_{\Omega}|\omega(y)||y|^{\theta} \mathrm{d} y<\infty .
$$

Finally, for any $\lambda \in(0,1), C^{\lambda}(\bar{\Omega})$ (resp., $C^{\lambda, 0}\left(\overline{Q_{T}}\right)$ ) is the space of all the functions $\omega \in \mathcal{B}(\bar{\Omega})$ (resp., $\omega \in \mathcal{B}\left(\overline{Q_{T}}\right)$ ) which are uniformly Hölder continuous in $y$ with exponent $\lambda$ on $\bar{\Omega}$ (resp., on $\overline{Q_{T}}$ ). $B_{r}(y)$ will denote the open 
ball in $\mathbb{R}^{2}$ with centre $y$ and radius $r$. At any point $y \in \partial \Omega(=\partial B), n=\left(n_{1}, n_{2}\right)$ will denote the unit outer normal vector to $\partial \Omega($ i.e. $n=-y)$, and $\tau=\left(\tau_{1}, \tau_{2}\right)$ will denote the unit tangent vector $\tau=y^{\perp}=\left(-y_{2}, y_{1}\right)$. For any scalar-valued function $\omega, \operatorname{curl} \omega=\left(\partial \omega / \partial y_{2},-\partial \omega / \partial y_{1}\right)$ and $\nabla \omega=\left(\partial \omega / \partial y_{1}, \partial \omega / \partial y_{2}\right)$. For any vector-valued function $v=\left(v_{1}, v_{2}\right), \operatorname{curl} v=\partial v_{2} / \partial y_{1}-\partial v_{1} / \partial y_{2}, \operatorname{div} \varphi=\partial v_{1} / \partial y_{1}+\partial v_{2} / \partial y_{2}$ and $\nabla v=\left(\partial v_{i} / \partial y_{j}\right)_{1 \leqslant i, j \leqslant 2}$. The main result in this paper is the following one.

Theorem 1.1. Let $\theta>0,0<\lambda<1, a \in \mathcal{B}(\bar{\Omega}) \cap H^{1}(\Omega)$ and $b \in \mathbb{R}^{2}$. Assume that $\operatorname{div} a=0,(a-b) \cdot n_{\left.\right|_{\partial B}}=0$, $\lim _{|y| \rightarrow+\infty} a(y)=0$, and curl $a \in L_{\theta}^{1}(\Omega) \cap C^{\lambda}(\bar{\Omega})$. Then there exists a solution $(v, q, l)$ of $(1.8)-(1.13)$ such that $v, \frac{\partial v}{\partial t}, \nabla v, \nabla q \in \mathcal{B}\left(\overline{Q_{T}}\right), v \in C^{1}\left([0, T], L^{2}(\Omega)\right) \cap C\left([0, T], H^{1}(\Omega)\right), q \in C\left([0, T], \widehat{H}^{1}(\Omega)\right)$ and $l \in C^{1}([0, T])$. Such a solution is unique up to an arbitrary function of $t$ which may be added to $q$.

Remark 1.2. We may notice that, with the above regularity, the solution $v$ satisfies the following property

$$
\lim _{|y| \rightarrow \infty} v(y, t)=0
$$

uniformly with respect to $t \in[0, T]$.

In the above theorem, we have denoted by $\widehat{H}^{1}(\Omega)$ the homogeneous Sobolev space

$$
\widehat{H}^{1}(\Omega)=\left\{q \in L_{l o c}^{2}(\bar{\Omega}) \mid \nabla q \in L^{2}(\Omega)\right\}
$$

where $q \in L_{\text {loc }}^{2}(\bar{\Omega})$ means that $q \in L^{2}\left(\Omega \cap B_{0}\right)$ for any open ball $B_{0} \subset \mathbb{R}^{2}$ with $B_{0} \cap \Omega \neq \emptyset$.

The kinetic energy of the system is given by

$$
E(t)=\frac{1}{2} m|l(t)|^{2}+\frac{1}{2} \int_{\Omega}|v(y, t)|^{2} \mathrm{~d} y .
$$

A great role will be played in the sequel by the scalar vorticity $\omega=\operatorname{curl} v$, which turns out to be bounded in $L^{1}(\Omega) \cap L^{\infty}(\Omega)$. (Notice that the initial vorticity $\omega_{0}:=\operatorname{curl} a$ clearly belongs to $L^{1}(\Omega) \cap L^{\infty}(\Omega)$.) Using the smoothness of the solution provided by Theorem 1.1, it is very easy to obtain the following result.

Corollary 1.3. Let $(v, l)$ be as in Theorem 1.1. Then the quantities $E(t)$ and $\|\omega(t)\|_{L^{p}(\Omega)}($ for any $p \in[1,+\infty])$ remain constant.

A large part of the proof of Theorem 1.1 relies on the machinery developed in [22] to prove the existence of classical solutions to Euler system in an exterior domain. However, unlike [22], a fixed-point argument cannot be applied directly to Euler system, due to a lack of pressure estimate. On the other hand, when we compare the assumptions of our main result to those required in [22], we note that

(1) no additional assumption has to be made here in order to insure the uniqueness of the solution;

(2) the initial velocity $a$ has to belong to $H^{1}(\Omega)$.

The intrusion of an $L^{2}$-estimate in a classical theory may look awkward at first sight. It is nevertheless necessary, as the boundedness of the speed of the rigid ball cannot be obtained without the aid of the energy conservation for the system solid+fluid. Thus, a feature of the problem investigated here is that we need estimates both in $L^{\infty}$ and in $L^{2}$.

To prove Theorem 1.1 we proceed in three steps. In the first step, we construct a strong solution of an approximated system in which Euler equations has been replaced by Navier-Stokes equations (with suitable boundary conditions). In the second step, we demonstrate that the vorticity associated with the strong solution of Navier-Stokes system is bounded in $L^{\infty}(\Omega) \cap L_{\theta}^{1}(\Omega)$, uniformly with respect to the viscosity coefficient. These estimates, combined with a standard energy estimate, provide the velocity estimates needed to pass to the limit. In the final step, we let the viscosity coefficient tend to 0 , and we prove that the solution to (1.8)-(1.13) has the regularity depicted in Theorem 1.1. 
The paper is outlined as follows. In Section 2 some preliminary results are given together with their proof. Section 3 is devoted to the construction of strong solutions to Navier-Stokes approximated system. The energy and vorticity estimates are established in Section 4. Finally, the proof of Theorem 1.1 is given in Section 5.

\section{Preliminaries}

\subsection{Extension of the velocity field to the plane}

Let $\rho:=m / \pi$ denote the (uniform) density of the rigid body. In the system (1.8)-(1.13), we can extend $v$ to $\mathbb{R}^{2}$ by taking $v(y, t)=l(t)$ for all $y \in B$ and all $t \geqslant 0$. Then $\operatorname{div} v=0$ in $\mathbb{R}^{2} \times[0, T]$. This suggests us to introduce the following spaces:

$$
\begin{gathered}
\mathcal{H}=\left\{\phi \in L^{2}\left(\mathbb{R}^{2}\right) \mid \operatorname{div}(\phi)=0 \text { in } \mathbb{R}^{2}, \quad \nabla \phi=0 \text { in } B\right\}, \\
\mathcal{V}=\left\{\phi \in \mathcal{H} \mid \phi_{\mid \Omega} \in H^{1}(\Omega)\right\} .
\end{gathered}
$$

We also define a scalar product in $L^{2}\left(\mathbb{R}^{2}\right)$ which is equivalent to the usual one:

$$
(u, v)_{\rho}:=\int_{\Omega} u \cdot v \mathrm{~d} x+\rho \int_{B} u \cdot v \mathrm{~d} x .
$$

The spaces $L^{2}\left(\mathbb{R}^{2}\right)$ and $\mathcal{H}$ are obviously Hilbert spaces for the scalar product $(., .)_{\rho}$. On the other hand, for any $u \in \mathcal{H}$ there exists a unique $l_{u} \in \mathbb{R}^{2}$ such that $u=l_{u}$ in $B$. It follows that for all $u, v \in \mathcal{H}$

$$
(u, v)_{\rho}=\int_{\Omega} u \cdot v \mathrm{~d} x+m l_{u} \cdot l_{v} .
$$

The space $\mathcal{V}$ is also a Hilbert space for the scalar product

$$
(u, v)_{\mathcal{V}}:=(u, v)_{\rho}+\int_{\Omega} \nabla u: \nabla v \mathrm{~d} x
$$

A first technical result is the following

Lemma 2.1. Let $u, v \in \mathcal{V}$, and let $k, l \in \mathbb{R}^{2}$ denote the constants such that $v=k$ and $u=l$ in B. Suppose that $u_{\mid \Omega} \in H^{2}(\Omega)$ and that $\operatorname{curl} u=0$ on $\partial B$. Then, we have the following identity

$$
\int_{\partial B} v \cdot \frac{\partial u}{\partial n} \mathrm{~d} \Gamma=\int_{\partial B}(v-k) \cdot(u-l) \mathrm{d} \Gamma,
$$

where $u$ and $v$ on $\partial B$ stand respectively for the traces of the functions $u_{\mid \Omega} \in H^{1}(\Omega)$ and $v_{\mid \Omega} \in H^{1}(\Omega)$.

Proof. Since $\operatorname{div} u=0$ and $\operatorname{div} v=0$ in $\mathcal{D}^{\prime}\left(\mathbb{R}^{2}\right)$, we have that

$$
(u-l) \cdot n=(v-k) \cdot n=0 \text { on } \partial B .
$$

By using the above equations, we deduce that

$$
(v-k) \cdot \nabla[(u-l) \cdot n]=0 \text { on } \partial B .
$$

On the other hand, since $\operatorname{curl} u=0$ on $\partial B$, we easily obtain that

$$
\nabla[(u-l) \cdot n]=\frac{\partial u}{\partial n}-(u-l) \text { on } \partial B,
$$


which implies that

$$
(v-k) \cdot \nabla[(u-l) \cdot n]=(v-k) \cdot \frac{\partial u}{\partial n}-(v-k) \cdot(u-l) \text { on } \partial B .
$$

The above equation and (2.4) imply that

$$
\int_{\partial B} v \cdot \frac{\partial u}{\partial n} \mathrm{~d} \Gamma=k \cdot \int_{\partial B} \frac{\partial u}{\partial n} \mathrm{~d} \Gamma+\int_{\partial B}(v-k) \cdot(u-l) \mathrm{d} \Gamma .
$$

Now, since $\operatorname{div} u=0$ and $\operatorname{curl} u=0$ on $\partial B$, we have that

$$
\int_{\partial B} \frac{\partial u}{\partial n} \mathrm{~d} \Gamma=\left(\int_{\partial B} \frac{\partial u}{\partial \tau} \mathrm{d} \Gamma\right)^{\perp}=0
$$

where $\tau=-n^{\perp}$. The above relation and (2.5) yield the result.

On the other hand, by using Hölder's inequality and a classical Sobolev embedding theorem, we have the following result.

Lemma 2.2. For all

$$
v, w \in L^{2}\left(0, T ; H^{2}(\Omega)\right) \cap L^{\infty}\left(0, T ; H^{1}(\Omega)\right),
$$

we have $(w \cdot \nabla) v \in L^{5 / 2}\left(0, T ; L^{2}(\Omega)\right)$ and

$$
\|(w \cdot \nabla) v\|_{L^{5 / 2}\left(0, T ; L^{2}(\Omega)\right)} \leqslant C\|w\|_{L^{\infty}\left(0, T ; H^{1}(\Omega)\right)}\|v\|_{L^{\infty}\left(0, T ; H^{1}(\Omega)\right)}^{1 / 5}\|v\|_{L^{2}\left(0, T ; H^{2}(\Omega)\right)}^{4 / 5},
$$

where $C$ is a positive constant depending only on $\Omega$.

\subsection{Velocity versus vorticity}

As it is well known, any $L^{p}$-norm of the vorticity is left unchanged by Euler flows. This is no longer true for Navier-Stokes flows, but we shall see that such norms remain bounded. Such vorticity estimates will result in velocity estimates thanks to the following result, which relates the velocity to the vorticity, the speed of the rigid body and the circulation of the flow along $\partial B$.

Proposition 2.3. Let $l \in \mathbb{R}^{2}, C \in \mathbb{R}$ and $\omega \in L^{1}(\Omega) \cap L^{\infty}(\Omega)$. Then there exists a unique vector field $v \in \mathcal{B}(\bar{\Omega})$ fulfilling

$$
\begin{array}{rlrl}
\operatorname{curl} v & =\omega & & \text { in } \Omega \\
\operatorname{div} v & =0 & & \text { in } \Omega \\
v \cdot n=l \cdot n & & \text { on } \partial B \\
\text { and } \quad & \lim _{\partial B} v \cdot \tau \mathrm{d} \Gamma & =C & \\
& \lim _{|y| \rightarrow+\infty} v(y) & =0 . &
\end{array}
$$

Furthermore, $v \in L^{p}(\Omega) \forall p \in(2,+\infty], \nabla v \in L^{p}(\Omega) \forall p \in(1,+\infty)$ and there exist some positive constants $K_{p}, K_{p}^{\prime}$ such that

$$
\begin{aligned}
\|v\|_{L^{p}(\Omega)} & \leqslant K_{p}\left(|l|+|C|+\|\omega\|_{L^{1}(\Omega)}+\|\omega\|_{L^{\infty}(\Omega)}\right) \quad \forall p \in(2,+\infty] \\
\|\nabla v\|_{L^{p}(\Omega)} & \leqslant K_{p}^{\prime}\left(|l|+|C|+\|\omega\|_{L^{p}(\Omega)}\right) \quad \forall p \in(1,+\infty) .
\end{aligned}
$$


Proof.

First Step. Existence of a vector field $v \in \mathcal{B}(\bar{\Omega})$ fulfilling $(2.7)-(2.11)$.

We first see that we may restrict ourselves to the situation where $l=0$ and $C=0$.

(i) Reduction to the case $l=0$.

We need the following lemma.

Lemma 2.4. Let $l \in \mathbb{R}^{2}$. Then there exists a vector field $d_{1} \in C^{\infty}\left(\mathbb{R}^{2}, \mathbb{R}^{2}\right)$ such that $\operatorname{div} d_{1}=0$ on $\mathbb{R}^{2}$ and

$$
d_{1}(y)= \begin{cases}l & \text { if }|y| \leqslant 2 \\ 0 & \text { if }|y| \geqslant 3\end{cases}
$$

Proof of Lemma 2.4. It is sufficient to pick any function $\theta \in C_{0}^{\infty}\left(\mathbb{R}^{2}\right)$ such that

$$
\theta(y)= \begin{cases}1 & \text { if }|y| \leqslant 2 \\ 0 & \text { if }|y| \geqslant 3\end{cases}
$$

and to set $d_{1}(y):=\operatorname{curl}\left(\theta(y) y \cdot l^{\perp}\right)$, where $l^{\perp}:=\left(-l_{2}, l_{1}\right)$.

Setting $v_{1}:=v-d_{1}$, we see that $(2.7)-(2.11)$ is changed into

$$
\begin{aligned}
\operatorname{curl} v_{1}=\omega_{1}:=\omega-\operatorname{curl} d_{1} & \text { in } \Omega \\
\operatorname{div} v_{1}=0 & \text { in } \Omega \\
v_{1} \cdot n=0 & \text { on } \partial B \\
\text { and } \quad \lim _{|y| \rightarrow+\infty} v_{1} \cdot \tau \mathrm{d} \Gamma=C & \\
& v_{1}(y)=0 .
\end{aligned}
$$

Notice that $\omega_{1} \in L^{1}(\Omega) \cap L^{\infty}(\Omega)$, as $\operatorname{curl} d_{1} \in C_{0}^{\infty}\left(\mathbb{R}^{2}\right)$.

(ii) Reduction to the case $C=0$.

We need the following lemma.

Lemma 2.5. There exists a vector field $d_{2} \in C^{\infty}\left(\bar{\Omega}, \mathbb{R}^{2}\right)$ such that $\operatorname{div} d_{2}=0, d_{2}(y)=0$ for $|y| \geqslant 3, d_{2} \cdot n=0$ for $|y|=1$ and $\int_{\partial B} d_{2} \cdot \tau \mathrm{d} \Gamma=1$.

Proof of Lemma 2.5. It is sufficient to pick any function $h \in C^{\infty}([1,+\infty), \mathbb{R})$, such that

$$
h(r)= \begin{cases}(2 \pi)^{-1} & \text { if } r \leqslant 2 \\ 0 & \text { if } r \geqslant 3\end{cases}
$$

and to set $d_{2}(y)=h(|y|) y^{\perp}$.

The change of unknown function

$$
v_{2}:=v_{1}-C d_{2}
$$

transforms (2.14)-(2.18) into

$$
\begin{aligned}
\operatorname{curl} v_{2}=\omega_{2}:=\omega_{1}-C \operatorname{curl} d_{2} & \text { in } \Omega \\
\operatorname{div} v_{2}=0 & \text { in } \Omega \\
v_{2} \cdot n=0 & \text { on } \partial B \\
\int_{\partial B} v_{2} \cdot \tau \mathrm{d} \Gamma=0 & \\
\text { and } \quad \lim _{|y| \rightarrow+\infty} v_{2}(y)=0 . &
\end{aligned}
$$


Notice that $\omega_{2} \in L^{1}(\Omega) \cap L^{\infty}(\Omega)$, because $d_{2}(y)=0$ for $|y| \geqslant 3$.

Second Step. Construction of a solution to (2.19)-(2.23).

Let $G(x, y)$ denotes the Green function for the exterior zero-Dirichlet problem for $-\Delta$, so that a solution $u(y)$ of

is given by

$$
\left\{\begin{aligned}
-\Delta u=f & \text { in } \Omega \\
u=0 & \text { on } \partial B
\end{aligned}\right.
$$

$$
u(y)=\frac{1}{2 \pi} \int_{\Omega} G(y, z) f(z) \mathrm{d} z .
$$

According to ([22], Lem. 1.3 and (2.27) p. 80), the vector field

$$
\bar{v}_{2}(y):=\frac{1}{2 \pi} \int_{\Omega} \operatorname{curl}_{y} G(y, z) \omega_{2}(z) \mathrm{d} z
$$

belongs to $\mathcal{B}(\bar{\Omega})$ and it satisfies (2.19)-(2.23), except possibly (2.22). By ([22], Lem. 1.5), there exists a vector field $\tilde{v}_{2} \in \mathcal{B}(\bar{\Omega})$ fulfilling

$$
\begin{aligned}
\operatorname{curl} \tilde{v}_{2}=0 & \text { in } \Omega \\
\operatorname{div} \tilde{v}_{2}=0 & \text { in } \Omega \\
\tilde{v}_{2} \cdot n=0 & \text { on } \partial B \\
\int_{\partial B} \tilde{v}_{2} \cdot \tau \mathrm{d} \Gamma=\int_{\partial B} \bar{v}_{2} \cdot \tau \mathrm{d} \Gamma & \\
\text { and } \lim _{|y| \rightarrow+\infty} \tilde{v}_{2}(y)=0 . &
\end{aligned}
$$

Then $v_{2}:=\bar{v}_{2}-\tilde{v}_{2}$ belongs to $\mathcal{B}(\bar{\Omega})$ and satisfies (2.19)-(2.23). It follows that (2.7)-(2.11) has at least one solution $v \in \mathcal{B}(\bar{\Omega})$. The uniqueness of such a solution follows from ([22], Lem. 2.14). (Notice that the hypothesis $V \in C^{1}(\Omega)$ in the statement of this lemma may easily be dropped.)

Third Step. The $L^{p}$ - estimates (2.12)-(2.13).

The idea is to extend $v$ as a solenoidal vector fluid on $\mathbb{R}^{2}$ and to use the classical $L^{p}$ estimates for such vector fields. We need the following result.

Lemma 2.6. Let $E(\bar{\Omega})=\{v \in \mathcal{B}(\bar{\Omega}) ; \operatorname{div} v=0$ on $\Omega$ and $v \cdot n=0$ on $\partial B\}, E\left(\mathbb{R}^{2}\right)=\left\{v \in \mathcal{B}\left(\mathbb{R}^{2}\right) ; \operatorname{div} v=0\right\}$, and $D=\left\{y \in \mathbb{R}^{2} ; 1<|y|<2\right\}$. Then there exists an extension operator $\Lambda: E(\bar{\Omega}) \rightarrow E\left(\mathbb{R}^{2}\right)\left(i . e ., \Lambda(v)_{\left.\right|_{\Omega}}=v\right)$ fulfilling the following property. For any $p \in(2,+\infty)$ there exists a constant $c_{p}>0$ such that for any $v \in$ $E(\bar{\Omega}) \cap W^{1, p}(D)$, its extension $\bar{v}=\Lambda(v)$ belongs to $W^{1, p}\left(B_{2}(0)\right)$ and satisfies

$$
\|\bar{v}\|_{W^{1, p}\left(B_{2}(0)\right)} \leqslant c_{p}\|v\|_{W^{1, p}(D)} .
$$

Proof of Lemma 2.6. Pick any $v \in E(\bar{\Omega})$ and set $w\left(y_{1}, y_{2}\right):=v\left(-y_{2}, y_{1}\right)$. Then it is easily seen that $\operatorname{curl} w(y)=$ $\operatorname{div} v\left(y^{\perp}\right)=0$ and that $\int_{\partial B} w \cdot \tau \mathrm{d} \Gamma=-\int_{\partial B} v \cdot n \mathrm{~d} \Gamma=0$. We infer from ([22], Lem. 2.13), that there exists a (unique) function $\varphi \in C^{1}(\bar{\Omega})$ such that $\varphi(1,0)=0$ and $w=\nabla \varphi$. Let $\psi\left(y_{1}, y_{2}\right):=\varphi\left(y_{2},-y_{1}\right)$ for all $y=\left(y_{1}, y_{2}\right) \in \bar{\Omega}$. An easy calculation yields $\operatorname{curl} \psi=v$. The proof of next result is left to the reader.

Lemma 2.7. There exists an extension operator $\Gamma: C^{1}(\bar{\Omega}) \rightarrow C^{1}\left(\mathbb{R}^{2}\right)$ fulfilling the following property. For any $p \in(2,+\infty)$ there exists a constant $c_{p}^{\prime}>0$ such that for any $\psi \in C^{1}(\bar{\Omega}) \cap W^{2, p}(D)$, its extension $\bar{\psi}=\Gamma(\psi)$ belongs to $W^{2, p}\left(B_{2}(0)\right)$ and satisfies

$$
\|\bar{\psi}\|_{W^{2, p}\left(B_{2}(0)\right)} \leqslant c_{p}^{\prime}\|\psi\|_{W^{2, p}(D)}
$$


Let $\bar{\psi}=\Gamma(\psi)$ be as given in Lemma 2.7 and set $\bar{v}=\operatorname{curl} \bar{\psi}$. Clearly, $\bar{v}_{\left.\right|_{\bar{\Omega}}}=v, \operatorname{div} \bar{v}=0$ and $\bar{v} \in \mathcal{B}\left(\mathbb{R}^{2}\right)$. Assume now that $v \in W^{1, p}(D)$ for some $p>2$. $C$ denoting a constant (depending only on $p$ ) which may vary from line to line, we have

$$
\begin{aligned}
\|\bar{v}\|_{W^{1, p}\left(B_{2}(0)\right)} & \leqslant C\|\bar{\psi}\|_{W^{2, p}\left(B_{2}(0)\right)} \\
& \leqslant C\|\psi \psi \mid\|_{W^{2, p}(D)} \\
& \leqslant C\|\varphi\| \|_{W^{2, p}(D)} \\
& \leqslant C\|w\|_{W^{1, p}(D)} \\
& \leqslant C\|v\|_{W^{1, p}(D)}
\end{aligned}
$$

$$
\begin{aligned}
& (\text { as } \bar{v}=\operatorname{curl} \bar{\psi}) \\
& (\text { by }(2.30)) \\
& \left(\text { as } \psi\left(y_{1}, y_{2}\right)=\varphi\left(y_{2},-y_{1}\right)\right) \\
& \left(\text { as }\|\nabla \varphi\|_{L^{\infty}(D)} \leqslant C\|w\|_{W^{1, p}(D)}\right) \\
& \left(\text { as } w\left(y_{1}, y_{2}\right)=v\left(-y_{2}, y_{1}\right)\right) .
\end{aligned}
$$

This completes the proof of Lemma 2.6.

Let $\bar{v}=\Lambda(v)$ be the extension of the solution $v \in \mathcal{B}(\bar{\Omega})$ to $(2.7)-(2.11)$ given by Lemma 2.6. We claim that $v \in W^{1, p}(D)$ for any $p>2$. Indeed, we may write

$$
v(y)=v_{2}(y)+d_{1}(y)+C d_{2}(y) \quad \forall y \in D
$$

where $d_{1}, d_{2} \in C^{\infty}(\bar{D})$ and $v_{2} \in \mathcal{B}(\bar{\Omega})$ solves (2.19)-(2.23). As in the proof of Lemma 2.6 we infer the existence of a function $\psi_{2} \in C^{1}(\bar{\Omega})$ such that $v_{2}=\operatorname{curl} \psi_{2}$ and $\psi_{2}=0$ on $\partial B$ (by (2.21)). As in addition $-\Delta \psi_{2}=$ $\operatorname{curl} v_{2}=\omega_{2} \in L^{\infty}(\Omega) \cap L^{1}(\Omega)$, we obtain (see, for instance, [13]) that $\psi_{2} \in W_{l o c}^{2, p}(\Omega)$ for any $p<\infty$, and consequently that $\psi_{2} \in W^{2, p}(D)$. Therefore, $v_{2} \in W^{1, p}(D)$, and we infer from Lemma 2.6 that $\bar{v}_{2} \in W_{l o c}^{1, p}\left(\mathbb{R}^{2}\right)$. Let $\bar{\omega}_{2}:=\operatorname{curl} \bar{v}_{2} \in L_{l o c}^{p}\left(\mathbb{R}^{2}\right)$. As $\left(\bar{\omega}_{2}\right)_{\left.\right|_{\Omega}}=\omega_{2}$ and $\omega_{2} \in L^{1}(\Omega) \cap L^{\infty}(\Omega)$, we conclude that $\bar{\omega}_{2} \in L^{1}\left(\mathbb{R}^{2}\right) \cap L^{p}\left(\mathbb{R}^{2}\right)$ for all $p<+\infty$. According to ([12], Lem. 2.1), the function

$$
\tilde{v}_{2}(y)=\frac{1}{2 \pi} \int_{\mathbb{R}^{2}} \frac{(y-z)^{\perp}}{|y-z|^{2}} \bar{\omega}_{2}(z) \mathrm{d} z
$$

satisfies $\tilde{v}_{2} \in L^{p}\left(\mathbb{R}^{2}\right) \quad \forall p \in(2,+\infty]$ and $\nabla \tilde{v}_{2} \in L^{p}\left(\mathbb{R}^{2}\right) \quad \forall 1<p<+\infty$ (hence $\tilde{v}_{2} \in \mathcal{B}\left(\mathbb{R}^{2}\right)$ and $\lim _{|y| \rightarrow+\infty} \tilde{v}_{2}(y)=0$.) Furthermore, $\operatorname{div} \tilde{v}_{2}=0$, and $\operatorname{curl} \tilde{v}_{2}=\bar{\omega}_{2}$. A slight modification of ([22], Lem. 2.14), shows that $\tilde{v}_{2}$ and $\bar{v}_{2}$ agree. This completes the proof of Proposition 2.3.

\section{NAVIER-Stokes APPROXIMATION FOR THE FLUID}

In order to solve (1.8)-(1.13), we follow the idea of P.L. Lions ([24], pp. 128-136), for the Euler equations. We first introduce the following system where we have replaced Euler equations by Navier-Stokes equations (with suitable boundary conditions).

$$
\begin{aligned}
\frac{\partial v}{\partial t}+(v \cdot \nabla) v-(l \cdot \nabla) v-\nu \Delta v+\nabla q=0, & \text { in } \Omega \times[0, T], \\
\operatorname{div} v=0, & \text { in } \Omega \times[0, T], \\
v \cdot n=l \cdot n, & \text { on } \partial B \times[0, T], \\
\operatorname{curl} v=0, & \text { on } \partial B \times[0, T], \\
m l^{\prime}=\int_{\partial B} q n \mathrm{~d} \Gamma, & \text { in }[0, T], \\
v(y, 0)=a(y), & y \in \Omega, \\
l(0)=b . &
\end{aligned}
$$

The system (3.1)-(3.7) will allow us to solve the original problem by passing to the limit when $\nu \rightarrow 0$. A similar problem with viscosity, but with Dirichlet boundary conditions, was studied in [31]. 


\subsection{Study of the linearised problem}

The first step to solve (3.1)-(3.7) is to consider the linearised problem:

$$
\begin{aligned}
\frac{\partial v}{\partial t}-\nu \Delta v+\nabla q=f, & \text { in } \Omega \times[0, T], \\
\operatorname{div} v=0, & \text { in } \Omega \times[0, T], \\
v \cdot n=l \cdot n, & \text { on } \partial B \times[0, T], \\
\operatorname{curl} v=0, & \text { on } \partial B \times[0, T], \\
m l^{\prime}=\int_{\partial B} q n \mathrm{~d} \Gamma, & \text { in }[0, T], \\
v(y, 0)=a(y), & y \in \Omega, \\
l(0)=b . &
\end{aligned}
$$

In order to solve (3.8)-(3.14) we use a semigroup approach. Let us define

$$
D(A)=\left\{\phi \in \mathcal{H} \mid \phi_{\mid \Omega} \in H^{2}(\Omega), \quad \operatorname{curl} \phi=0 \text { on } \partial B\right\}
$$

and the operators

$$
\mathcal{A} \phi=\left\{\begin{array}{ll}
-\nu \Delta \phi & \text { in } \Omega, \\
0 & \text { in } B,
\end{array} \quad \forall \phi \in D(A)\right.
$$

and

$$
A \phi=\mathbb{P} \mathcal{A} \phi, \quad \forall \phi \in D(A),
$$

where $\mathbb{P}$ denotes the orthogonal projector from $L^{2}\left(\mathbb{R}^{2}\right)$ (endowed with the $(.,.) \rho$ scalar product) onto $\mathcal{H}(\mathcal{H}$ is clearly a closed subspace of $\left.L^{2}\left(\mathbb{R}^{2}\right)\right)$. In order to solve (3.8)-(3.14), we write the linearised system in the form

$$
v(t) \in D(A), \quad v^{\prime}+A v=\mathbb{P} f, \quad v(0)=v_{0} .
$$

We give in the following proposition some important properties of the operator $A$ :

Proposition 3.1. There exists $\lambda>0$ such that the operator $-(\lambda I+A)$ is self-adjoint and m-dissipative. Moreover, for any $u \in D(A)$, we have that

$$
\|u\|_{H^{2}(\Omega)} \leqslant C\|(\lambda I+A) u\|_{\rho} .
$$

Proof. Let $u, v \in D(A)$. We have that

$$
(A u, v)_{\rho}=(\mathcal{A} u, v)_{\rho}=-\nu \int_{\Omega} \Delta u \cdot v \mathrm{~d} y=\nu \int_{\Omega} \nabla u: \nabla v \mathrm{~d} y-\nu \int_{\partial B} v \cdot \frac{\partial u}{\partial n} \mathrm{~d} \Gamma .
$$

Therefore, by using Lemma 2.1, we obtain that

$$
(A u, v)_{\rho}=\nu \int_{\Omega} \nabla u: \nabla v \mathrm{~d} y-\nu \int_{\partial B}(v-k) \cdot(u-l) \mathrm{d} \Gamma
$$

where $k, l \in \mathbb{R}^{2}$ are such that $v=k$ and $u=l$ in $B$. It follows that $A$ is symmetric.

Moreover, for all $u \in D(A)$, we have

$$
(A u, u)_{\rho}=\nu \int_{\Omega}|\nabla u|^{2} \mathrm{~d} y-\nu \int_{\partial B}|u-l|^{2} \mathrm{~d} \Gamma .
$$


Using the following estimate (see e.g. the proof of [1], Lem. IX.9)

$$
\int_{\partial B}|v|^{2} \mathrm{~d} \Gamma \leqslant C\left(\int_{\Omega}|v|^{2} \mathrm{~d} y\right)^{1 / 2}\left(\int_{\Omega}|\nabla v|^{2} \mathrm{~d} y\right)^{1 / 2} \quad \forall v \in H^{1}(\Omega)
$$

we also have that

$$
\begin{aligned}
\int_{\partial B}|u-l|^{2} \mathrm{~d} \Gamma & \leqslant 4 \pi|l|^{2}+2 \int_{\partial B}|u|^{2} \mathrm{~d} \Gamma \\
& \leqslant 4 \int_{B}|u|^{2} \mathrm{~d} y+C_{1} \int_{\Omega}|u|^{2} \mathrm{~d} y+\frac{1}{2} \int_{\Omega}|\nabla u|^{2} \mathrm{~d} y .
\end{aligned}
$$

The above relation, combined with (3.21), implies that for some constant $C_{2}=C_{2}(\nu)>0$ and for all $u \in D(A)$,

$$
((\lambda I+A) u, u)_{\rho} \geqslant \lambda\|u\|_{\rho}^{2}+\frac{\nu}{2} \int_{\Omega}|\nabla u|^{2} \mathrm{~d} y-C_{2}\|u\|_{\rho}^{2} .
$$

Consequently, for $\lambda>C_{2}$, the operator $\lambda I+A$ is (strictly) positive.

By using Lax-Milgram's lemma, we deduce that for all $f \in \mathcal{H}$, there exists a unique $u \in \mathcal{V}$ such that

$$
\nu \int_{\Omega} \nabla u: \nabla v \mathrm{~d} y-\nu \int_{\partial B}(v-k) \cdot(u-l) \mathrm{d} \Gamma+\lambda(u, v)_{\rho}=(f, v)_{\rho}, \quad \forall v \in \mathcal{V} .
$$

In particular, (3.24) holds if

$$
v= \begin{cases}\phi & \text { in } \Omega, \\ 0 & \text { in } B\end{cases}
$$

where $\phi$ is any $C^{\infty}$ function with compact support in $\Omega$ and such that $\operatorname{div}(\phi)=0$. Then it follows from ([32], Prop. 1.1), that there exists $p \in \mathcal{D}^{\prime}(\Omega)$ such that

$$
\lambda u-\nu \Delta u+\nabla p=f \quad \text { in } \mathcal{D}^{\prime}(\Omega) .
$$

Since $u \in \mathcal{V}$, we have that $\operatorname{div}(u)=0$ in $\Omega$ and that there exists $l \in \mathbb{R}^{2}$ with

$$
u(y)=l \quad \forall y \in B .
$$

The above relations imply that $u$ satisfies the Stokes type system:

$$
\begin{aligned}
\lambda u-\nu \Delta u+\nabla p=f & \text { in } \Omega, \\
\operatorname{div} u=0 & \text { in } \Omega, \\
u \cdot n=l \cdot n & \text { on } \partial B, \\
\operatorname{curl} u=0 & \text { on } \partial B .
\end{aligned}
$$

Thus, by a regularity result for elliptic equations, we have that $u_{\mid \Omega} \in H^{2}(\Omega), p \in \widehat{H}^{1}(\Omega)$ and

$$
\|u\|_{H^{2}(\Omega)} \leqslant C\|f\|_{L^{2}(\Omega)} .
$$

Finally, from (3.15) and (3.24), we have that $u \in D(A)$ and $(\lambda I+A) u=f$. Therefore, $-(\lambda I+A)$ is a m-dissipative operator, which gives the result. 
An important consequence of Proposition 3.1 is the following.

Corollary 3.2. Let $f \in L^{2}\left(0, T ; L^{2}(\Omega)\right)$ and $a \in H^{1}(\Omega)$ be such that

$$
\begin{array}{cl}
\operatorname{div} a=0, & \text { in } \Omega, \\
a \cdot n=b \cdot n, & \text { on } \partial B .
\end{array}
$$

Then the system (3.8)-(3.14) admits a unique solution $(v, q, l)$ with

$$
\begin{gathered}
v \in L^{2}\left(0, T ; H^{2}(\Omega)\right) \cap C\left([0, T] ; H^{1}(\Omega)\right) \cap H^{1}\left(0, T ; L^{2}(\Omega)\right), \\
q \in L^{2}\left(0, T ; \widehat{H}^{1}(\Omega)\right), \quad l \in H^{1}\left(0, T ; \mathbb{R}^{2}\right) .
\end{gathered}
$$

Moreover, there exists a positive constant $K$ such that

$$
\begin{array}{r}
\|v\|_{L^{2}\left(0, T ; H^{2}(\Omega)\right)}+\|v\|_{L^{\infty}\left(0, T ; H^{1}(\Omega)\right)}+\|v\|_{H^{1}\left(0, T ; L^{2}(\Omega)\right)}+\|\nabla q\|_{L^{2}\left(0, T ; L^{2}(\Omega)\right)} \\
+\|l\|_{H^{1}\left(0, T ; \mathbb{R}^{2}\right)} \leqslant K\left(\|a\|_{H^{1}(\Omega)}+|b|+\|f\|_{L^{2}\left(0, T ; L^{2}(\Omega)\right)}\right) .
\end{array}
$$

The constant $K$ depends only on $\Omega$ and $T$ and is non-decreasing with respect to $T$.

Proof. If we extend the functions $a$ and $f$ to $\mathbb{R}^{2}$ by

$$
\begin{aligned}
& a(y)=b \quad \forall y \in B, \\
& f(y)=0 \quad \forall y \in B,
\end{aligned}
$$

then we have that $a \in \mathcal{V}$ and $f \in L^{2}\left(0, T ; L^{2}\left(\mathbb{R}^{2}\right)\right)$. Therefore the Cauchy problem

$$
\begin{aligned}
v^{\prime}+A v & =\mathbb{P} f, \\
v(0) & =a
\end{aligned}
$$

admits a unique solution

$$
v \in L^{2}(0, T ; D(A)) \cap C\left([0, T] ; D\left((\lambda I+A)^{1 / 2}\right)\right) \cap H^{1}(0, T ; \mathcal{H}) .
$$

Since $v \in H^{1}(0, T ; \mathcal{H})$, there exists $l \in H^{1}\left(0, T ; \mathbb{R}^{2}\right)$, such that $v(y, t)=l(t)$ for all $y \in B$ and for all $t \in[0, T]$.

Let $\phi \in \mathcal{H}$. By taking the inner product of (3.27) by $\phi$, we obtain

$$
\left(v^{\prime}(t), \phi\right)_{\rho}+(\mathcal{A} v(t), \phi)_{\rho}=(f(t), \phi)_{\rho}
$$

for almost all $t \in[0, T]$. Thus

$$
\int_{\Omega} v^{\prime}(t) \cdot \phi \mathrm{d} y+m l^{\prime}(t) \cdot l_{\phi}+\int_{\Omega}(-\nu \Delta v) \cdot \phi \mathrm{d} y=\int_{\Omega} f(t) \cdot \phi \mathrm{d} y,
$$

for all $\phi \in \mathcal{H}$ and for almost all $t \in[0, T]$. In particular, (3.30) holds if

$$
\phi= \begin{cases}\psi & \text { in } \Omega \\ 0 & \text { in } B\end{cases}
$$

where $\psi$ is any $C^{\infty}$ function with compact support in $\Omega$ and such that $\operatorname{div}(\psi)=0$. For such $\phi,(3.30)$ gives

$$
\int_{\Omega}\left(v^{\prime}(t)-\nu \Delta v(t)-f(t)\right) \cdot \psi \mathrm{d} y=0,
$$


for any $C^{\infty}$ function $\psi$ with compact support in $\Omega$ and such that $\operatorname{div}(\psi)=0$. By ([32], Props. 1.1 and 1.2), there exists $q \in L^{2}\left(0, T ; \widehat{H}^{1}(\Omega)\right)$, such that

$$
v^{\prime}(t)-\nu \Delta v(t)+\nabla q(t)=f(t), \quad \text { in } \Omega .
$$

Hence we have proved that $v$ satisfies (3.8). Moreover, the above relation implies that

$$
\int_{\Omega}\left(v^{\prime}(t)-\nu \Delta v(t)+\nabla q(t)-f(t)\right) \cdot \phi \mathrm{d} y=0, \quad \forall \phi \in \mathcal{H},
$$

which yields

$$
\int_{\Omega}\left(v^{\prime}(t)-\nu \Delta v(t)-f(t)\right) \cdot \phi \mathrm{d} y=-\int_{\partial B} q(t) n \cdot \phi \mathrm{d} \Gamma, \quad \forall \phi \in \mathcal{H} .
$$

The above equality and (3.30) imply that

$$
m l^{\prime}(t) \cdot l_{\phi}=\int_{\partial B} q(t) n \cdot \phi \mathrm{d} \Gamma, \quad \forall \phi \in \mathcal{H},
$$

from which we deduce that

$$
m l^{\prime}(t)=\int_{\partial B} q(t) n \mathrm{~d} \Gamma .
$$

Hence $(v, q, l)$ is a solution of the system (3.8)-(3.14).

In order to prove the uniqueness of the solution to (3.8)-(3.14), it is sufficient to notice that the solutions of (3.8)-(3.14) satisfy the system (3.27)-(3.28), whose solutions are unique.

\subsection{Strong solutions for the nonlinear problem}

We go back to the nonlinear problem (3.1)-(3.7). In this subsection, we prove the following proposition:

Proposition 3.3. Suppose that $T>0, a \in H^{1}(\Omega)$, and that

$$
\begin{gathered}
\operatorname{div} a=0 \quad \text { in } \Omega, \\
a \cdot n=b \cdot n \quad \text { on } \partial B .
\end{gathered}
$$

Then, there exists a unique strong solution $(v, q, l)$ of (3.1)-(3.7) satisfying

$$
\begin{gathered}
v \in L^{2}\left(0, T ; H^{2}(\Omega)\right) \cap C\left([0, T] ; H^{1}(\Omega)\right) \cap H^{1}\left(0, T ; L^{2}(\Omega)\right), \\
q \in L^{2}\left(0, T ; \widehat{H}^{1}(\Omega)\right), \quad l \in H^{1}\left(0, T ; \mathbb{R}^{2}\right) .
\end{gathered}
$$

Proof.

First Step. Local in time existence.

We consider the application $\mathcal{Z}$ defined by

$$
\begin{aligned}
\mathcal{Z}: L^{2}\left(0, T ; L^{2}(\Omega)\right) & \rightarrow L^{2}\left(0, T ; L^{2}(\Omega)\right) \\
f & \mapsto-(v \cdot \nabla) v+(l \cdot \nabla) v
\end{aligned}
$$

where $(v, p, l)$ is the solution of $(3.8)-(3.14)$. Let

$$
B(0, R)=\left\{v \in L^{2}\left(0, T ; L^{2}(\Omega)\right) \mid\|v\|_{L^{2}\left(0, T ; L^{2}(\Omega)\right)} \leqslant R\right\} .
$$


By using Lemma 2.2 and Corollary 3.2, we have that

$$
\|\mathcal{Z}(f)\|_{L^{5 / 2}\left(0, T ; L^{2}(\Omega)\right)} \leqslant C\left(\|a\|_{H^{1}(\Omega)}+|b|+\|f\|_{L^{2}\left(0, T ; L^{2}(\Omega)\right)}\right)^{2} .
$$

Hence, by Hölder's inequality, $\mathcal{Z}$ maps $L^{2}\left(0, T ; L^{2}(\Omega)\right)$ into itself and

$$
\|\mathcal{Z}(f)\|_{L^{2}\left(0, T ; L^{2}(\Omega)\right)} \leqslant T^{1 / 10} C\left(\|a\|_{H^{1}(\Omega)}+|b|+\|f\|_{L^{2}\left(0, T ; L^{2}(\Omega)\right)}\right)^{2} .
$$

Therefore for $R>\|a\|_{H^{1}(\Omega)}+|b|$ and $T \leqslant\left(\frac{1}{4 C R}\right)^{10}, \mathcal{Z}$ maps $B(0, R)$ into $B(0, R)$.

Similarly, we can prove that $\left.\mathcal{Z}\right|_{B(0, R)}$ is a contraction for $T$ small enough. This demonstrates that there exists a unique (strong) solution $v$ of $(3.1)-(3.7)$ on some interval $\left[0, T^{\prime}\right]$ with $T^{\prime} \leq T$.

Second Step. Global in time existence.

In this part of the proof, $C$ will denote a positive constant (depending only on $\|a\|_{H^{1}(\Omega)},|b|, m, T$, and $\nu$ ), which may vary from line to line.

To see that the solution $v$ obtained above may be extended to $[0, T]$, it is sufficient to prove that there exists some positive constant $K=K\left(\|a\|_{H^{1}(\Omega)},|b|, m, T, \nu\right)$ such that $\|v(t)\|_{H^{1}(\Omega)} \leqslant K$ for all $t \in\left[0, T^{\prime}\right]$.

By taking the inner product of $(3.27)$ by $v(t) \in \mathcal{H}$ (where $f$ denotes the function $-(v \cdot \nabla) v+(l \cdot \nabla) v$ extended by 0 on $B)$, we obtain that

$$
\frac{1}{2} \frac{\mathrm{d}}{\mathrm{d} t}\|v(t)\|_{\rho}^{2}+(v(t), A v(t))_{\rho}=(\mathbb{P} f(t), v(t))_{\rho}, \quad \text { a.e. in }\left(0, T^{\prime}\right) .
$$

Since

and

$$
\int_{\Omega}[(v \cdot \nabla) v] \cdot v \mathrm{~d} y=\frac{1}{2} \int_{\partial B}|v|^{2}(v \cdot n) \mathrm{d} \Gamma
$$

we get that

$$
v(y, t) \cdot n=l(t) \cdot n \quad \forall y \in \partial B
$$

$$
(f(t), v(t))_{\rho}=\int_{\Omega}[-(v(t) \cdot \nabla) v(t)+(l(t) \cdot \nabla) v(t)] \cdot v(t) \mathrm{d} y=0, \quad \text { a.e. in }\left(0, T^{\prime}\right) .
$$

Therefore, by integrating (3.31) with respect to $t$, we obtain that

$$
\|v(t)\|_{\rho}^{2}+2 \int_{0}^{t}(v(s), A v(s))_{\rho} \mathrm{d} s \leqslant\|v(0)\|_{\rho}^{2}, \quad \forall t \in\left[0, T^{\prime}\right]
$$

and thus that

$$
\|v(t)\|_{\rho}^{2} \leqslant 2 \lambda \int_{0}^{t}\|v(s)\|_{\rho}^{2} \mathrm{~d} s+\|v(0)\|_{\rho}^{2}, \quad \forall t \in\left[0, T^{\prime}\right]
$$

where $\lambda$ is defined in Proposition 3.1. The above relation combined with Gronwall's inequality implies that

$$
\|v(t)\|_{\rho}^{2}=\|v(t)\|_{L^{2}(\Omega)}^{2}+m|l(t)|^{2} \leqslant C \quad \forall t \in\left[0, T^{\prime}\right]
$$

The above relation, (3.32) and (3.23) imply that

$$
\begin{aligned}
\int_{0}^{t}\|v(s)\|_{H^{1}(\Omega)}^{2} \mathrm{~d} s & \leqslant \frac{2}{\nu} \int_{0}^{t}(v(s),(\lambda I+A) v(s))_{\rho} \mathrm{d} s+\int_{0}^{t}\|v(s)\|_{L^{2}(\Omega)}^{2} \mathrm{~d} s \\
& \leqslant C \quad \forall t \in\left[0, T^{\prime}\right] .
\end{aligned}
$$

On the other hand, by taking the inner product of $(3.27)$ by $A v(t) \in \mathcal{H}$, we obtain that

$$
\left(v^{\prime}(t), A v(t)\right)_{\rho}+(A v(t), A v(t))_{\rho}=(\mathbb{P} f(t), A v(t))_{\rho}, \quad \text { a.e. in }\left(0, T^{\prime}\right) .
$$


The above relation yields that

$$
\begin{aligned}
\frac{1}{2} \frac{\mathrm{d}}{\mathrm{d} t}(v(t), A v(t))_{\rho}+\|A v(t)\|_{\rho}^{2}= & \int_{\Omega} f(t) \cdot A v(t) \mathrm{d} y \\
= & -\int_{\Omega}[(v(t) \cdot \nabla) v(t)] \cdot(A v(t)) \mathrm{d} y+\int_{\Omega}[(l(t) \cdot \nabla) v(t)] \cdot(A v(t)) \mathrm{d} y \\
\leqslant & C\|v(t)\|_{L^{2}(\Omega)}^{1 / 2}\|v(t)\|_{H^{1}(\Omega)}\|v(t)\|_{H^{2}(\Omega)}^{3 / 2} \\
& +\|(l(t) \cdot \nabla) v(t)\|_{L^{2}(\Omega)}\|v(t)\|_{H^{2}(\Omega)} \quad \text { a.e. in }\left(0, T^{\prime}\right) .
\end{aligned}
$$

From Proposition 3.1,

$$
\|v\|_{H^{2}(\Omega)} \leqslant C\|(\lambda I+A) v\|_{\rho}
$$

which clearly gives

$$
c^{\prime}\|v\|_{H^{2}(\Omega)}^{2} \leqslant\|A v\|_{\rho}^{2}+\|v\|_{\rho}^{2}
$$

for some positive constant $c^{\prime}=c^{\prime}(\nu)$. Relations (3.36), (3.37) and Young's inequality imply that

$$
\begin{aligned}
\frac{1}{2} \frac{\mathrm{d}}{\mathrm{d} t}(v(t), A v(t))_{\rho}+c^{\prime}\|v(t)\|_{H^{2}(\Omega)}^{2} \leqslant & C\|v(t)\|_{L^{2}(\Omega)}^{2}\|v(t)\|_{H^{1}(\Omega)}^{4}+\frac{c^{\prime}}{4}\|v(t)\|_{H^{2}(\Omega)}^{2}+C\|(l(t) \cdot \nabla) v(t)\|_{L^{2}(\Omega)}^{2} \\
& +\frac{c^{\prime}}{4}\|v(t)\|_{H^{2}(\Omega)}^{2}+\|v(t)\|_{\rho}^{2}, \quad \text { a.e. in }\left(0, T^{\prime}\right) .
\end{aligned}
$$

By (3.34), we have that

$$
\|(l(t) \cdot \nabla) v(t)\|_{L^{2}(\Omega)}^{2} \leqslant C\|v(t)\|_{H^{1}(\Omega)}^{2}, \quad\|v(t)\|_{L^{2}(\Omega)}^{2} \leqslant C
$$

thus, (3.38) implies that

$$
\frac{\mathrm{d}}{\mathrm{d} t}(v(t), A v(t))_{\rho}+c^{\prime}\|v(t)\|_{H^{2}(\Omega)}^{2} \leqslant C\left(\|v(t)\|_{H^{1}(\Omega)}^{4}+\|v(t)\|_{H^{1}(\Omega)}^{2}+1\right) \quad \text { a.e. in }\left(0, T^{\prime}\right) .
$$

If we integrate the above relation with respect to $t$, we get by (3.21) that for all $t \in\left[0, T^{\prime}\right]$,

$$
(v(t), A v(t))_{\rho}+c^{\prime} \int_{0}^{t}\|v(s)\|_{H^{2}(\Omega)}^{2} \mathrm{~d} s \leqslant C\left(1+\int_{0}^{t}\left[\|v(s)\|_{H^{1}(\Omega)}^{2}+1\right]\|v(s)\|_{H^{1}(\Omega)}^{2} \mathrm{~d} s\right) .
$$

The above relation, (3.34) and (3.23) yield that for all $t \in\left[0, T^{\prime}\right]$,

$$
\frac{\nu}{2}\|v(t)\|_{H^{1}(\Omega)}^{2}+c^{\prime} \int_{0}^{t}\|v(s)\|_{H^{2}(\Omega)}^{2} \mathrm{~d} s \leqslant C\left(1+\int_{0}^{t}\left[\|v(s)\|_{H^{1}(\Omega)}^{2}+1\right]\|v(s)\|_{H^{1}(\Omega)}^{2} \mathrm{~d} s\right)
$$

which, combined to Gronwall's lemma and to (3.35), gives that

$$
\|v(t)\|_{H^{1}(\Omega)}^{2} \leqslant \frac{C}{\nu} \exp \left(\frac{C}{\nu}\right)=: K^{2} \quad \forall t \in\left[0, T^{\prime}\right]
$$

The proof of Proposition 3.3 is complete. 


\section{Some estimates for the Navier-Stokes Problem}

From now on we denote by $\left(v_{\nu}, q_{\nu}, l_{\nu}\right)$ the solution of (3.1)-(3.7) corresponding to the value $\nu$ of the viscosity coefficient. The goal of this section is to establish some estimates for the strong solutions of the Navier-Stokes equations

$$
\begin{aligned}
\frac{\partial v_{\nu}}{\partial t}+\left(v_{\nu} \cdot \nabla\right) v_{\nu}-\left(l_{\nu} \cdot \nabla\right) v_{\nu}-\nu \Delta v_{\nu}+\nabla q_{\nu}=0, & \text { in } \Omega \times[0, T], \\
\operatorname{div} v_{\nu}=0, & \text { in } \Omega \times[0, T], \\
v_{\nu} \cdot n=l_{\nu} \cdot n, & \text { on } \partial B \times[0, T], \\
\operatorname{curl} v_{\nu}=0, & \text { on } \partial B \times[0, T], \\
m l_{\nu}^{\prime}=\int_{\partial B} q_{\nu} n \Gamma, & \text { in }[0, T], \\
v_{\nu}(y, 0)=a(y), & y \in \Omega, \\
l_{\nu}(0)=b . &
\end{aligned}
$$

Recall that the corresponding vorticity $\omega_{\nu}$ is defined by

$$
\omega_{\nu}=\operatorname{curl} v_{\nu}
$$

From Proposition 3.3, we have that for any $b \in \mathbb{R}^{2}$ and any $a \in H^{1}(\Omega)$ such that

$$
\operatorname{div} a=0 \text { in } \Omega \text { and } a \cdot n=b \cdot n \quad \text { on } \partial B
$$

there exists a unique strong solution $\left(v_{\nu}, l_{\nu}, q_{\nu}\right)$ of (4.1)-(4.7) satisfying:

$$
\begin{gathered}
v_{\nu} \in L^{2}\left(0, T ; H^{2}(\Omega)\right) \cap C\left([0, T] ; H^{1}(\Omega)\right) \cap H^{1}\left(0, T ; L^{2}(\Omega)\right), \\
q_{\nu} \in L^{2}\left(0, T ; \widehat{H}^{1}(\Omega)\right), \quad l_{\nu} \in H^{1}\left(0, T ; \mathbb{R}^{2}\right) .
\end{gathered}
$$

\subsection{Energy estimates}

Now, we prove an energy estimate for the Navier-Stokes model (4.1)-(4.7).

Proposition 4.1. Let $b \in \mathbb{R}^{2}$ and let $a \in H^{1}(\Omega)$ be a function satisfying

$$
\operatorname{div} a=0 \text { in } \Omega \text { and } a \cdot n=b \cdot n \quad \text { on } \partial B .
$$

Then there exists a positive constant $C$, independent on $\nu$, such that the unique solution $\left(v_{\nu}, l_{\nu}, q_{\nu}\right)$ of $(4.1)-(4.7)$ satisfies

$$
\int_{\Omega}\left|v_{\nu}(y, t)\right|^{2} \mathrm{~d} y+m\left|l_{\nu}(t)\right|^{2} \leqslant \mathrm{e}^{C \nu T}\left[\int_{\Omega}|a(y)|^{2} \mathrm{~d} y+m|b(t)|^{2}\right] \quad \forall t \in[0, T] .
$$

Proof. By multiplying (4.1) by $v_{\nu}$ and integrating over $\Omega \times(0, t)$ we obtain

$$
\int_{0}^{t} \int_{\Omega} \frac{\partial v_{\nu}}{\partial t} \cdot v_{\nu} \mathrm{d} y \mathrm{~d} s+\int_{0}^{t} \int_{\Omega}\left[\left(\left(v_{\nu}-l_{\nu}\right) \cdot \nabla\right) v_{\nu}\right] \cdot v_{\nu} \mathrm{d} y \mathrm{~d} s-\nu \int_{0}^{t} \int_{\Omega} \Delta v_{\nu} \cdot v_{\nu} \mathrm{d} y \mathrm{~d} s+\int_{0}^{t} \int_{\Omega} \nabla q_{\nu} \cdot v_{\nu} \mathrm{d} y \mathrm{~d} s=0 .
$$

Performing integrations by parts and using Lemma 2.1, we easily obtain that

$$
\int_{\Omega}\left|v_{\nu}(y, t)\right|^{2} \mathrm{~d} y+m\left|l_{\nu}(t)\right|^{2}+2 \nu \int_{0}^{t} \int_{\Omega}\left|\nabla v_{\nu}\right|^{2} \mathrm{~d} y \mathrm{~d} s \leqslant \int_{\Omega}|a(y)|^{2} \mathrm{~d} y+m|b|^{2}+2 \nu \int_{0}^{t} \int_{\partial B}\left|v_{\nu}-l_{\nu}\right|^{2} \mathrm{~d} \Gamma \mathrm{d} s
$$


and by using (3.22), we have that

$$
\int_{\Omega}\left|v_{\nu}(y, t)\right|^{2} \mathrm{~d} y+m\left|l_{\nu}(t)\right|^{2} \leqslant\left[\int_{\Omega}|a(y)|^{2} \mathrm{~d} y+m|b|^{2}\right]+C \nu \int_{0}^{t}\left[\left(\int_{\Omega}\left|v_{\nu}\right|^{2} \mathrm{~d} y\right)+m\left|l_{\nu}\right|^{2}\right] \mathrm{d} s .
$$

Therefore, by applying Gronwall's Lemma, the above equation yields that for every $t \in[0, T]$

$$
\int_{\Omega}\left|v_{\nu}(y, t)\right|^{2} \mathrm{~d} y+m\left|l_{\nu}(t)\right|^{2} \leqslant \mathrm{e}^{C \nu t}\left[\int_{\Omega}|a(y)|^{2} \mathrm{~d} y+m|b|^{2}\right]
$$

which completes the proof.

\subsection{Vorticity estimates}

Now, we aim to establish some estimates for the vorticity

$$
\omega_{\nu}=\operatorname{curl} v_{\nu}
$$

which satisfies the following system

$$
\begin{aligned}
\frac{\partial \omega_{\nu}}{\partial t}+\left(\left(v_{\nu}-l_{\nu}\right) \cdot \nabla\right) \omega_{\nu}-\nu \Delta \omega_{\nu}=0, & \text { in } \Omega \times[0, T], \\
\omega_{\nu}=0, & \text { on } \partial B \times[0, T], \\
\omega_{\nu}(y, 0)=\omega_{0}(y), & y \in \Omega,
\end{aligned}
$$

where $\omega_{0}:=\operatorname{curl} a$. We have the following estimate.

Proposition 4.2. Let $\omega_{0} \in L^{1}(\Omega) \cap L^{\infty}(\Omega)$. Then for all $p \in[1,+\infty]$ and for all $t \in[0, T]$ we have that

$$
\left\|\omega_{\nu}(t)\right\|_{L^{p}(\Omega)} \leqslant\left\|\omega_{0}\right\|_{L^{p}(\Omega)} .
$$

Proof. We can note that, since

$$
v_{\nu} \in L^{2}\left(0, T ; H^{2}(\Omega)\right) \cap C\left([0, T] ; H^{1}(\Omega)\right) \cap H^{1}\left(0, T ; L^{2}(\Omega)\right)
$$

we have that

$$
\omega_{\nu} \in L^{2}\left(0, T ; H^{1}(\Omega)\right) \cap C\left([0, T] ; L^{2}(\Omega)\right) \cap H^{1}\left(0, T ; H^{-1}(\Omega)\right)
$$

and therefore

$$
\frac{\partial \omega_{\nu}}{\partial t} \in L^{2}\left(0, T ; H^{-1}(\Omega)\right) \quad \text { and } \quad\left(v_{\nu}-l_{\nu}\right) \cdot \nabla \omega_{\nu} \in L^{2}\left(0, T ; H^{-1}(\Omega)\right) .
$$

Then multiplying (4.12) by $\varphi \in L^{2}\left(0, T, H_{0}^{1}(\Omega)\right)$ and integrating with respect to time, we have that

$$
0=\int_{0}^{t}\left\langle\frac{\partial \omega_{\nu}}{\partial t}, \varphi\right\rangle_{H^{-1} \times H_{0}^{1}} \mathrm{~d} s+\int_{0}^{t}\left\langle\left(\left(v_{\nu}-l_{\nu}\right) \cdot \nabla\right) \omega_{\nu}, \varphi\right\rangle_{H^{-1} \times H_{0}^{1}} \mathrm{~d} s-\nu \int_{0}^{t}\left\langle\Delta \omega_{\nu}, \varphi\right\rangle_{H^{-1} \times H_{0}^{1}} \mathrm{~d} s .
$$

The above equation easily yields that

$$
0=\int_{0}^{t}\left\langle\frac{\partial \omega_{\nu}}{\partial t}, \varphi\right\rangle_{H^{-1} \times H_{0}^{1}}^{\mathrm{d} s}+\int_{0}^{t}\left\langle\operatorname{div}\left(\left(v_{\nu}-l_{\nu}\right) \omega_{\nu}\right), \varphi\right\rangle_{H^{-1} \times H_{0}^{1}} \mathrm{~d} s+\nu \int_{0}^{t} \int_{\Omega} \nabla \omega_{\nu} \cdot \nabla \varphi \mathrm{d} y \mathrm{~d} s .
$$


Now, we analyse each integral term and consider two cases:

Case $1(p \geqslant 2)$.

As the function $\left|\omega_{\nu}\right|^{p-2} \omega_{\nu}$ cannot be a priori taken as a test function, we are led to truncate it. (This method often proves to be useful when establishing a priori estimates, see e.g. [25].) Let $R>0$ and let $T_{R}$ denote the function

We consider the following test function

$$
T_{R}(r)= \begin{cases}R & \text { if } r>R \\ r & \text { if }-R \leqslant r \leqslant R, \\ -R & \text { if } r<-R .\end{cases}
$$

$$
\varphi=\left|T_{R}\left(\omega_{\nu}\right)\right|^{p-2} T_{R}\left(\omega_{\nu}\right) \in L^{2}\left(0, T, H_{0}^{1}(\Omega)\right) \cap L^{\infty}\left(Q_{T}\right) .
$$

Simple calculations yield

$$
\nabla \varphi=(p-1)\left|\omega_{\nu}\right|^{p-2}\left(\nabla \omega_{\nu}\right) 1_{\left|\omega_{\nu}\right| \leqslant R}
$$

and therefore, we have that

$$
\begin{aligned}
\int_{0}^{t}\left\langle\operatorname{div}\left(\left(v_{\nu}-l_{\nu}\right) \omega_{\nu}\right), \varphi\right\rangle_{H^{-1} \times H_{0}^{1}} \mathrm{~d} s & =(1-p) \int_{0}^{t} \int_{\left|\omega_{\nu}\right| \leqslant R}\left(\left(v_{\nu}-l_{\nu}\right) \omega_{\nu}\left|\omega_{\nu}\right|^{p-2}\right) \cdot \nabla \omega_{\nu} \mathrm{d} y \mathrm{~d} s \\
& =-\frac{p-1}{p} \int_{0}^{t} \int_{\Omega}\left(v_{\nu}-l_{\nu}\right) \cdot \nabla\left(\left|T_{R}\left(\omega_{\nu}\right)\right|^{p}\right) \mathrm{d} y \mathrm{~d} s=0
\end{aligned}
$$

by (4.2) and (4.13). Moreover, we have that

$$
\nu \int_{0}^{t} \int_{\Omega} \nabla \omega_{\nu} \nabla \varphi \mathrm{d} y \mathrm{~d} s=\nu(p-1) \int_{0}^{t} \int_{\left|\omega_{\nu}\right| \leqslant R}\left|\nabla \omega_{\nu}\right|^{2}\left|\omega_{\nu}\right|^{p-2} \mathrm{~d} y \mathrm{~d} s
$$

Now, let us define the function

$$
F_{R}(r)=\int_{0}^{r}\left|T_{R}(\sigma)\right|^{p-2} T_{R}(\sigma) \mathrm{d} \sigma .
$$

We can use this function to give another expression of the first term in (4.16):

$$
\int_{0}^{t}\left\langle\frac{\partial \omega_{\nu}}{\partial t}, \varphi\right\rangle_{H^{-1} \times H_{0}^{1}} \mathrm{~d} s=\int_{0}^{t}\left\langle\frac{\partial \omega_{\nu}}{\partial t}, F_{R}^{\prime}\left(\omega_{\nu}\right)\right\rangle_{H^{-1} \times H_{0}^{1}} \mathrm{~d} s .
$$

Thus, by using an approximating "regular" sequence for $\omega_{\nu}$, we have that

$$
\int_{0}^{t}\left\langle\frac{\partial \omega_{\nu}}{\partial t}, \varphi\right\rangle_{H^{-1} \times H_{0}^{1}} \mathrm{~d} s=\int_{\Omega} F_{R}\left(\omega_{\nu}(t)\right) \mathrm{d} y-\int_{\Omega} F_{R}\left(\omega_{0}\right) \mathrm{d} y .
$$

On the other hand, it is easy to see that

$$
F_{R}(r)=\frac{1}{p}\left|T_{R}(r)\right|^{p}+R^{p-1}(|r|-R)^{+} .
$$

By using (4.16), (4.17), (4.18) and (4.19), we have that

$$
\int_{\Omega} F_{R}\left(\omega_{\nu}(t)\right) \mathrm{d} y+\nu(p-1) \int_{0}^{t} \int_{\left|\omega_{\nu}\right| \leqslant R}\left|\nabla \omega_{\nu}\right|^{2}\left|\omega_{\nu}\right|^{p-2} \mathrm{~d} y \mathrm{~d} s=\int_{\Omega} F_{R}\left(\omega_{0}\right) \mathrm{d} y
$$


hence

$$
\int_{\Omega} F_{R}\left(\omega_{\nu}\right)(t) \mathrm{d} y \leqslant \int_{\Omega} F_{R}\left(\omega_{0}\right) \mathrm{d} y .
$$

Letting $R \nearrow+\infty$, we obtain by the monotone convergence theorem

$$
\frac{1}{p} \int_{\Omega}\left|\omega_{\nu}\right|^{p}(t) \leqslant \frac{1}{p} \int_{\Omega}\left|\omega_{0}\right|^{p},
$$

or equivalently, that for every $t \in[0, T]$

$$
\left\|\omega_{\nu}(t)\right\|_{L^{p}(\Omega)} \leqslant\left\|\omega_{0}\right\|_{L^{p}(\Omega)}(<\infty) .
$$

Letting $p \rightarrow+\infty$, we conclude that

for every $t \in[0, T]$.

$$
\left\|\omega_{\nu}(t)\right\|_{L^{\infty}(\Omega)} \leqslant\left\|\omega_{0}\right\|_{L^{\infty}(\Omega)}
$$

Case $2(1<p<2)$.

Now, we consider the case $1<p<2$. Let $h_{\varepsilon}(r):=(|r|+\varepsilon)^{p-2} r$ for all $r \in \mathbb{R}$. Proceeding in an analogous way to the previous case, we consider the test function

$$
\varphi=h_{\varepsilon}\left(\omega_{\nu}\right)=\left(\left|\omega_{\nu}\right|+\varepsilon\right)^{p-2} \omega_{\nu} \in L^{2}\left(0, T, H_{0}^{1}(\Omega)\right) \cap L^{\infty}\left(Q_{T}\right) .
$$

We define the function

which obviously fulfils

$$
H_{\varepsilon}(r)=\int_{0}^{r} h_{\varepsilon}(\sigma) \mathrm{d} \sigma
$$

$$
H_{\varepsilon}^{\prime}(r)=(|r|+\varepsilon)^{p-2} r .
$$

By multiplying (4.12) by $\varphi$ and integrating in time and space, we obtain that

$$
0=\int_{0}^{t}\left\langle\frac{\partial \omega_{\nu}}{\partial t}, \varphi\right\rangle_{H^{-1} \times H_{0}^{1}} \mathrm{~d} s+\int_{0}^{t}\left\langle\left(\left(v_{\nu}-l_{\nu}\right) \cdot \nabla\right) \omega_{\nu}, \varphi\right\rangle_{H^{-1} \times H_{0}^{1}} \mathrm{~d} s+\nu \int_{0}^{t} \int_{\Omega} \nabla \omega_{\nu} \cdot \nabla \varphi \mathrm{d} y \mathrm{~d} s .
$$

Firstly, we have that

$$
\begin{aligned}
\int_{0}^{t}\left\langle\frac{\partial \omega_{\nu}}{\partial t}, \varphi\right\rangle_{H^{-1} \times H_{0}^{1}} \mathrm{~d} s & =\int_{0}^{t}\left\langle\frac{\partial \omega_{\nu}}{\partial t}, H_{\varepsilon}^{\prime}\left(\omega_{\nu}\right)\right\rangle_{H^{-1} \times H_{0}^{1}} \mathrm{~d} s \\
& =\int_{\Omega} H_{\varepsilon}\left(\omega_{\nu}(y, t)\right) \mathrm{d} y-\int_{\Omega} H_{\varepsilon}\left(\omega_{0}(y)\right) \mathrm{d} y .
\end{aligned}
$$

On the other hand, we have that

$$
\begin{aligned}
\int_{\Omega} \nabla \omega_{\nu} \cdot \nabla \varphi \mathrm{d} y & =\int_{\Omega} \nabla \omega_{\nu} \cdot\left\{(p-2)\left(\left|\omega_{\nu}\right|+\varepsilon\right)^{p-3}\left|\omega_{\nu}\right| \nabla \omega_{\nu}+\left(\left|\omega_{\nu}\right|+\varepsilon\right)^{p-2} \nabla \omega_{\nu}\right\} \mathrm{d} y \\
& =\int_{\Omega}\left|\nabla \omega_{\nu}\right|^{2}\left(\left|\omega_{\nu}\right|+\varepsilon\right)^{p-3}\left\{(p-1)\left|\omega_{\nu}\right|+\varepsilon\right\} \mathrm{d} y \geqslant 0 .
\end{aligned}
$$

Finally, we have that

$$
\left\langle\left(\left(v_{\nu}-l_{\nu}\right) \cdot \nabla\right) \omega_{\nu}, \varphi\right\rangle_{H^{-1} \times H_{0}^{1}}=-\int_{\Omega}\left(\left(v_{\nu}-l_{\nu}\right) \omega_{\nu}\right) \cdot \nabla \varphi \mathrm{d} y=-\int_{\Omega}\left(v_{\nu}-l_{\nu}\right) \cdot \nabla G\left(\omega_{\nu}\right) \mathrm{d} y,
$$


where $G$ denotes the function

$$
G(r)=\int_{0}^{r} \sigma h_{\varepsilon}^{\prime}(\sigma) \mathrm{d} \sigma
$$

Thus, since $\operatorname{div} v_{\nu}=0$ and $G(0)=0$, we have that

$$
\left\langle\left(\left(v_{\nu}-l_{\nu}\right) \cdot \nabla\right) \omega_{\nu}, \varphi\right\rangle_{H^{-1} \times H_{0}^{1}}=0 .
$$

Gathering (4.21), (4.22), (4.23) and (4.25), we obtain that

$$
\int_{\Omega} H_{\varepsilon}\left(\omega_{\nu}(y, t)\right) \mathrm{d} y+\nu \int_{0}^{t} \int_{\Omega}\left|\nabla \omega_{\nu}\right|^{2}\left(\left|\omega_{\nu}\right|+\varepsilon\right)^{p-3}\left\{(p-1)\left|\omega_{\nu}\right|+\varepsilon\right\} \mathrm{d} y \mathrm{~d} s=\int_{\Omega} H_{\varepsilon}\left(\omega_{0}(y)\right) \mathrm{d} y,
$$

and therefore we have that

$$
\int_{\Omega} H_{\varepsilon}\left(\omega_{\nu}(y, t)\right) \mathrm{d} y \leqslant \int_{\Omega} H_{\varepsilon}\left(\omega_{0}(y)\right) \mathrm{d} y
$$

Taking the limit as $\varepsilon \searrow 0$, we obtain by the monotone convergence theorem that for every $t \in[0, T]$

$$
\frac{1}{p} \int_{\Omega}\left|\omega_{\nu}(y, t)\right|^{p} \mathrm{~d} y \leqslant \frac{1}{p} \int_{\Omega}\left|\omega_{0}(y)\right|^{p} \mathrm{~d} y
$$

and consequently, we have that

$$
\left\|\omega_{\nu}(t)\right\|_{L^{p}(\Omega)} \leqslant\left\|\omega_{0}\right\|_{L^{p}(\Omega)} .
$$

Taking the limit as $p \rightarrow 1$, we obtain that

$$
\left\|\omega_{\nu}(t)\right\|_{L^{1}(\Omega)} \leqslant\left\|\omega_{0}\right\|_{L^{1}(\Omega)},
$$

which completes the proof.

Proposition 4.3. Let $\omega_{0} \in L^{1}(\Omega) \cap L^{\infty}(\Omega)$ be a function such that

$$
\int_{\Omega}\left|\omega_{0}(y)\right||y|^{\theta} \mathrm{d} y<\infty
$$

for a positive constant $\theta>0$.

Then there exists a positive constant $C>0$, such that for all $t \in[0, T]$ we have that

$$
\int_{\Omega}\left|\omega_{\nu}(y, t)\right||y|^{\theta} \mathrm{d} y \leqslant \mathrm{e}^{C t} \int_{\Omega}\left|\omega_{0}(y)\right||y|^{\theta} \mathrm{d} y
$$

Proof. To prove this result we proceed as above by choosing a convenient test function.

Let $\theta>0$. According to Proposition 4.2 we have that $\omega_{\nu} \in L^{\infty}\left(0, T, L^{p}(\Omega)\right)$, for all $p \in[1, \infty]$. On the other hand, $\left\|\omega_{0}\right\|_{L_{\theta}^{p}(\Omega)}^{p}:=\int_{\Omega}\left|\omega_{0}(y)\right|^{p}|y|^{\theta} \mathrm{d} y<\infty$ for all $p \in(1,+\infty)$, as $\omega_{0} \in L_{\theta}^{1}(\Omega) \cap L^{\infty}(\Omega)$.

For every $\delta>0$, let $\psi_{\delta} \in C^{\infty}(\Omega)$ be the function defined by

$$
\psi_{\delta}(y)=\exp (-\delta|y|)
$$

Thus, we have that

and therefore we obtain that

$$
\nabla \psi_{\delta}(y)=-\delta \frac{y}{|y|} \psi_{\delta}(y)
$$

$$
\nabla\left\{|y|^{\theta} \psi_{\delta}(y)\right\}=\left\{\theta \frac{y}{|y|^{2}}-\delta \frac{y}{|y|}\right\}|y|^{\theta} \psi_{\delta}(y)
$$


and

$$
\Delta\left\{|y|^{\theta} \psi_{\delta}(y)\right\}=\left\{\frac{\theta^{2}}{|y|^{2}}-\frac{\delta(2 \theta+1)}{|y|}+\delta^{2}\right\}|y|^{\theta} \psi_{\delta}(y)
$$

Pick any $p \in(1,2)$ and any $\varepsilon>0$, and consider the test function $\phi$ defined as

$$
\phi(y, t)=\varphi(y, t)|y|^{\theta} \psi_{\delta}(y)=\left(\left|\omega_{\nu}(y, t)\right|+\varepsilon\right)^{p-2} \omega_{\nu}(y, t)|y|^{\theta} \psi_{\delta}(y) .
$$

(Recall that $\varphi=h_{\varepsilon}\left(\omega_{\nu}\right)$.) We have that

$$
\phi \in L^{\infty}\left(0, T ; L^{p}(\Omega)\right) \cap L^{2}\left(0, T ; H_{0}^{1}(\Omega)\right)
$$

and

$$
\nabla \phi=\left(\varepsilon+(p-1)\left|\omega_{\nu}\right|\right)\left(\left|\omega_{\nu}\right|+\varepsilon\right)^{p-3} \nabla \omega_{\nu}|y|^{\theta} \psi_{\delta}(y)+\left(\left|\omega_{\nu}\right|+\varepsilon\right)^{p-2} \omega_{\nu} \nabla\left\{|y|^{\theta} \psi_{\delta}\right\} .
$$

Then, by multiplying the vorticity equation (4.12) by $\phi$ we have that

$$
0=\int_{0}^{t}\left\langle\frac{\partial \omega_{\nu}}{\partial t}, \phi\right\rangle_{H^{-1} \times H_{0}^{1}} \mathrm{~d} s+\int_{0}^{t}\left\langle\left(\left(v_{\nu}-l_{\nu}\right) \cdot \nabla\right) \omega_{\nu}, \phi\right\rangle_{H^{-1} \times H_{0}^{1}} \mathrm{~d} s+\nu \int_{0}^{t} \int_{\Omega} \nabla \omega_{\nu} \cdot \nabla \phi \mathrm{d} y \mathrm{~d} s
$$

As in the proof of Proposition 4.2, we study each one of the previous integrals. Analogously to the previous proof, we have that

$$
\int_{0}^{t}\left\langle\frac{\partial \omega_{\nu}}{\partial t}, \phi\right\rangle_{H^{-1} \times H_{0}^{1}} \mathrm{~d} s=\int_{\Omega} H_{\varepsilon}\left(\omega_{\nu}(y, t)\right)|y|^{\theta} \psi_{\delta}(y) \mathrm{d} y-\int_{\Omega} H_{\varepsilon}\left(\omega_{0}(y)\right)|y|^{\theta} \psi_{\delta}(y) \mathrm{d} y
$$

Notice that by (4.26)

$$
\int_{\Omega} H_{\varepsilon}\left(\omega_{\nu}(y, t)\right)|y|^{\theta} \psi_{\delta}(y) \mathrm{d} y \leqslant\left\||y|^{\theta} \psi_{\delta}(y)\right\|_{L^{\infty}(\Omega)} \int_{\Omega} H_{\varepsilon}\left(\omega_{\nu}(y, t)\right) \mathrm{d} y<\infty .
$$

On the other hand

$$
\begin{aligned}
\int_{\Omega} \nabla \omega_{\nu} \cdot \nabla \phi \mathrm{d} y=\int_{\Omega}\left(\varepsilon+(p-1)\left|\omega_{\nu}\right|\right)\left(\left|\omega_{\nu}\right|+\varepsilon\right)^{p-3}\left|\nabla \omega_{\nu}\right|^{2}|y|^{\theta} \psi_{\delta}(y) \mathrm{d} y & \\
& +\int_{\Omega}\left(\left|\omega_{\nu}\right|+\varepsilon\right)^{p-2} \omega_{\nu} \nabla \omega_{\nu} \cdot \nabla\left\{|y|^{\theta} \psi_{\delta}\right\} \mathrm{d} y
\end{aligned}
$$

The first integral term of the right-hand side of the above equation is non-negative and for the second one, since

$$
\left(\left|\omega_{\nu}\right|+\varepsilon\right)^{p-2} \omega_{\nu} \nabla \omega_{\nu}=\frac{1}{p} \nabla\left\{\left(\left|\omega_{\nu}\right|+\varepsilon\right)^{p}\right\}-\frac{\varepsilon}{p-1} \nabla\left\{\left(\left|\omega_{\nu}\right|+\varepsilon\right)^{p-1}\right\},
$$


we have that

$$
\begin{aligned}
\int_{\Omega}\left(\left|\omega_{\nu}\right|+\varepsilon\right)^{p-2} \omega_{\nu} \nabla \omega_{\nu} \cdot \nabla\left\{|y|^{\theta} \psi_{\delta}\right\} \mathrm{d} y= & \frac{1}{p} \int_{\Omega} \nabla\left\{\left(\left|\omega_{\nu}\right|+\varepsilon\right)^{p}\right\} \cdot \nabla\left\{|y|^{\theta} \psi_{\delta}\right\} \mathrm{d} y \\
& -\frac{\varepsilon}{p-1} \int_{\Omega} \nabla\left\{\left(\left|\omega_{\nu}\right|+\varepsilon\right)^{p-1}\right\} \cdot \nabla\left\{|y|^{\theta} \psi_{\delta}\right\} \mathrm{d} y \\
= & -\frac{1}{p} \int_{\Omega}\left(\left|\omega_{\nu}\right|+\varepsilon\right)^{p} \Delta\left\{|y|^{\theta} \psi_{\delta}\right\} \mathrm{d} y \\
& +\frac{\varepsilon}{p-1} \int_{\Omega}\left(\left|\omega_{\nu}\right|+\varepsilon\right)^{p-1} \Delta\left\{|y|^{\theta} \psi_{\delta}\right\} \mathrm{d} y+\left[\frac{2 \pi}{p(p-1)}(\theta-\delta) \mathrm{e}^{-\delta}\right] \varepsilon^{p}
\end{aligned}
$$

and thus, from (4.30), we have that

$$
\begin{aligned}
\int_{\Omega} \nabla \omega_{\nu} \cdot \nabla \phi \mathrm{d} y= & \int_{\Omega}\left(\varepsilon+(p-1)\left|\omega_{\nu}\right|\right)\left(\left|\omega_{\nu}\right|+\varepsilon\right)^{p-3}\left|\nabla \omega_{\nu}\right|^{2}|y|^{\theta} \psi_{\delta}(y) \mathrm{d} y \\
& -\frac{1}{p} \int_{\Omega}\left(\left|\omega_{\nu}\right|+\varepsilon\right)^{p}\left\{\frac{\theta^{2}}{|y|^{2}}-\frac{\delta(2 \theta+1)}{|y|}+\delta^{2}\right\}|y|^{\theta} \psi_{\delta}(y) \mathrm{d} y \\
& +\frac{\varepsilon}{p-1} \int_{\Omega}\left(\left|\omega_{\nu}\right|+\varepsilon\right)^{p-1}\left\{\frac{\theta^{2}}{|y|^{2}}-\frac{\delta(2 \theta+1)}{|y|}+\delta^{2}\right\}|y|^{\theta} \psi_{\delta}(y) \mathrm{d} y \\
& +\left[\frac{2 \pi}{p(p-1)}(\theta-\delta) \mathrm{e}^{-\delta}\right] \varepsilon^{p}
\end{aligned}
$$

Furthermore, we have that

$$
\begin{aligned}
\left\langle\left(\left(v_{\nu}-l_{\nu}\right) \cdot \nabla\right) \omega_{\nu}, \phi\right\rangle_{H^{-1} \times H_{0}^{1}} & =-\int_{\Omega}\left(v_{\nu}-l_{\nu}\right) \cdot \omega_{\nu} \nabla\left(\varphi|y|^{\theta} \psi_{\delta}\right) \mathrm{d} y \\
& =-\int_{\Omega} \omega_{\nu} \varphi\left(v_{\nu}-l_{\nu}\right) \cdot \nabla\left(|y|^{\theta} \psi_{\delta}\right) \mathrm{d} y-\int_{\Omega}|y|^{\theta} \psi_{\delta}\left(v_{\nu}-l_{\nu}\right) \cdot \omega_{\nu} \nabla \varphi \mathrm{d} y
\end{aligned}
$$

We study the right-hand side of the last equality and from (4.29) we have that

$$
\begin{aligned}
\int_{\Omega} \omega_{\nu} \varphi\left(v_{\nu}-l_{\nu}\right) \cdot \nabla\left(|y|^{\theta} \psi_{\delta}\right) \mathrm{d} y & =\int_{\Omega} \omega_{\nu}\left(\left|\omega_{\nu}\right|+\varepsilon\right)^{p-2} \omega_{\nu}\left(v_{\nu}-l_{\nu}\right) \cdot \nabla\left(|y|^{\theta} \psi_{\delta}\right) \mathrm{d} y \\
& =\int_{\Omega}\left\{\frac{\left|\omega_{\nu}\right|^{2}}{\left(\left|\omega_{\nu}\right|+\varepsilon\right)^{2}}\right\}\left(\left|\omega_{\nu}\right|+\varepsilon\right)^{p}\left(v_{\nu}-l_{\nu}\right) \cdot \nabla\left(|y|^{\theta} \psi_{\delta}\right) \mathrm{d} y \\
& =\int_{\Omega}\left\{\frac{\left|\omega_{\nu}\right|^{2}}{\left(\left|\omega_{\nu}\right|+\varepsilon\right)^{2}}\right\}\left(\left|\omega_{\nu}\right|+\varepsilon\right)^{p}|y|^{\theta} \psi_{\delta}(y)\left(v_{\nu}-l_{\nu}\right) \cdot\left\{\theta \frac{y}{|y|^{2}}-\delta \frac{y}{|y|}\right\} \mathrm{d} y
\end{aligned}
$$

The above equality implies that

$$
\left|\int_{\Omega} \omega_{\nu} \varphi\left(v_{\nu}-l_{\nu}\right) \cdot \nabla\left(|y|^{\theta} \psi_{\delta}\right) \mathrm{d} y\right| \leqslant\left\|v_{\nu}-l_{\nu}\right\|_{L^{\infty}(\Omega)}\{\theta+\delta\} \int_{\Omega}\left(\left|\omega_{\nu}\right|+\varepsilon\right)^{p}|y|^{\theta} \psi_{\delta} \mathrm{d} y
$$


For the second integral term, we proceed as in the proof of Proposition 4.2 (case $1<p<2$ ). More precisely, considering the function $G$ given in (4.24) we have that

$$
\begin{aligned}
\int_{\Omega}|y|^{\theta} \psi_{\delta}\left(v_{\nu}-l_{\nu}\right) \cdot \omega_{\nu} \nabla \varphi \mathrm{d} y & =\int_{\Omega}|y|^{\theta} \psi_{\delta}\left(v_{\nu}-l_{\nu}\right) \cdot \nabla\left[G\left(\omega_{\nu}\right)\right] \mathrm{d} y \\
& =-\int_{\Omega} \operatorname{div}\left\{|y|^{\theta} \psi_{\delta}\left(v_{\nu}-l_{\nu}\right)\right\} G\left(\omega_{\nu}\right) \mathrm{d} y \\
& =-\int_{\Omega} G\left(\omega_{\nu}\right)\left\{\left(v_{\nu}-l_{\nu}\right) \cdot \nabla\left\{|y|^{\theta} \psi_{\delta}\right\}\right\} \mathrm{d} y
\end{aligned}
$$

and therefore, gathering (4.37) and the above equation, we have that

$$
\begin{aligned}
\left\langle\left(\left(v_{\nu}-l_{\nu}\right) \cdot \nabla\right) \omega_{\nu}, \phi\right\rangle_{H^{-1} \times H_{0}^{1}}=-\int_{\Omega} \omega_{\nu} \varphi\left(v_{\nu}-l_{\nu}\right) & \cdot \nabla\left\{|y|^{\theta} \psi_{\delta}\right\} \mathrm{d} y \\
& +\int_{\Omega} G\left(\omega_{\nu}\right)|y|^{\theta} \psi_{\delta}(y)\left\{\left(v_{\nu}-l_{\nu}\right) \cdot\left\{\theta \frac{y}{|y|^{2}}-\delta \frac{y}{|y|}\right\}\right\} \mathrm{d} y .
\end{aligned}
$$

Then by replacing (4.32), (4.36) and (4.40) in (4.31), we obtain that

$$
\begin{aligned}
\int_{\Omega} H_{\varepsilon}\left(\omega_{\nu}(y, t)\right)|y|^{\theta} \psi_{\delta} \mathrm{d} y \leqslant & \int_{\Omega} H_{\varepsilon}\left(\omega_{0}\right)|y|^{\theta} \psi_{\delta} \mathrm{d} y+\left|\int_{0}^{t} \int_{\Omega} \omega_{\nu} \varphi\left(v_{\nu}-l_{\nu}\right) \cdot \nabla\left(|y|^{\theta} \psi_{\delta}\right) \mathrm{d} y \mathrm{~d} s\right| \\
& +\int_{0}^{t} \int_{\Omega}\left|G\left(\omega_{\nu}\right)\right||y|^{\theta} \psi_{\delta}\left|\left(v_{\nu}-l_{\nu}\right) \cdot\left\{\theta \frac{y}{|y|^{2}}-\delta \frac{y}{|y|}\right\}\right| \mathrm{d} y \mathrm{~d} s \\
& +\frac{\nu}{p} \int_{0}^{t} \int_{\Omega}\left(\left|\omega_{\nu}\right|+\varepsilon\right)^{p}|y|^{\theta} \psi_{\delta}\left|\frac{\theta^{2}}{|y|^{2}}-\frac{\delta(2 \theta+1)}{|y|}+\delta^{2}\right| \mathrm{d} y \mathrm{~d} s \\
& +\frac{\varepsilon \nu}{p-1} \int_{0}^{t} \int_{\Omega}\left(\left|\omega_{\nu}\right|+\varepsilon\right)^{p-1}|y|^{\theta} \psi_{\delta}\left|\frac{\theta^{2}}{|y|^{2}}-\frac{\delta(2 \theta+1)}{|y|}+\delta^{2}\right| \mathrm{d} y \mathrm{~d} s \\
& +\frac{2 \pi \nu}{p(p-1)}|\theta-\delta| \mathrm{e}^{-\delta} \varepsilon^{p} .
\end{aligned}
$$

Then from (4.39) we have that

$$
\begin{aligned}
& \int_{\Omega} H_{\varepsilon}\left(\omega_{\nu}(y, t)\right)|y|^{\theta} \psi_{\delta} \mathrm{d} y \leqslant \int_{\Omega} H_{\varepsilon}\left(\omega_{0}\right)|y|^{\theta} \psi_{\delta} \mathrm{d} y+\left\{C_{1}+\nu \frac{C_{2}}{p}\right\} \int_{0}^{t} \int_{\Omega}\left(\left|\omega_{\nu}\right|+\varepsilon\right)^{p}|y|^{\theta} \psi_{\delta} \mathrm{d} y \mathrm{~d} s \\
& \quad+C_{1} \int_{0}^{t} \int_{\Omega}\left|G\left(\omega_{\nu}\right)\right||y|^{\theta} \psi_{\delta} \mathrm{d} y \mathrm{~d} s+\frac{\varepsilon \nu C_{2}}{p-1} \int_{0}^{t} \int_{\Omega}\left(\left|\omega_{\nu}\right|+\varepsilon\right)^{p-1}|y|^{\theta} \psi_{\delta} \mathrm{d} y \mathrm{~d} s+\frac{2 \pi \nu}{p(p-1)}|\theta-\delta| \mathrm{e}^{-\delta} \varepsilon^{p}
\end{aligned}
$$

where

$$
C_{1}=(\theta+\delta)\left\|v_{\nu}-l_{\nu}\right\|_{L^{\infty}\left(Q_{T}\right)} \quad \text { and } \quad C_{2}=\theta^{2}+\delta(2 \theta+1)+\delta^{2} .
$$

The fact that $\left\|v_{\nu}-l_{\nu}\right\|_{L^{\infty}\left(Q_{T}\right)}$ remains bounded as $\nu \searrow 0$ readily follows from Propositions 2.3, 4.1 and 4.2 (see below Sect. 5.1.) Now, letting $\varepsilon \searrow 0$, we obtain that for some constant $C_{3}>0$

$$
\frac{1}{p} \int_{\Omega}\left|\omega_{\nu}(y, t)\right|^{p}|y|^{\theta} \psi_{\delta} \mathrm{d} y \leqslant \frac{1}{p} \int_{\Omega}\left|\omega_{0}\right|^{p}|y|^{\theta} \psi_{\delta} \mathrm{d} y+\left\{C_{1}+\nu \frac{C_{2}}{p}+C_{3}\right\} \int_{0}^{t} \int_{\Omega}\left|\omega_{\nu}\right|^{p}|y|^{\theta} \psi_{\delta} \mathrm{d} y \mathrm{~d} s
$$


Applying Gronwall's Lemma and then using the monotone convergence theorem in the limit $\delta \searrow 0$, we obtain that there exists a positive constant $C>0$, such that for all $t \in[0, T]$

$$
\int_{\Omega}\left|\omega_{\nu}(y, t)\right|^{p}|y|^{\theta} \mathrm{d} y \leqslant \mathrm{e}^{C t} \int_{\Omega}\left|\omega_{0}(y)\right|^{p}|y|^{\theta} \mathrm{d} y .
$$

Now, we have in the limit $p \rightarrow 1^{+}$

$$
\int_{\Omega}\left|\omega_{\nu}(y, t)\right||y|^{\theta} \mathrm{d} y \leqslant \mathrm{e}^{C t} \int_{\Omega}\left|\omega_{0}(y)\right||y|^{\theta} \mathrm{d} y \quad \forall t \in[0, T],
$$

and the proof is complete.

\section{Proof of Theorem 1.1}

\subsection{Passage to the limit}

It follows from (4.10) that $l_{\nu}$ is bounded in $L^{\infty}(0, T)$. On the other hand, the quantity $\int_{\partial B} v_{\nu} \cdot \tau \mathrm{d} \Gamma$ is also bounded in $L^{\infty}(0, T)$. Indeed, according to Stokes' theorem

$$
\int_{\partial B} v_{\nu} \cdot \tau \mathrm{d} \Gamma=-\int_{\Omega} \operatorname{curl} v_{\nu} \mathrm{d} y=-\int_{\Omega} \omega_{\nu} \mathrm{d} y
$$

hence, by (4.15),

$$
\left|\int_{\partial B} v_{\nu} \cdot \tau \mathrm{d} \Gamma\right| \leqslant\left\|\omega_{\nu}\right\|_{L^{1}(\Omega)} \leqslant\left\|\omega_{0}\right\|_{L^{1}(\Omega)}
$$

As $\omega_{\nu}$ is bounded in $L^{\infty}\left(0, T, L^{1}(\Omega) \cap L^{\infty}(\Omega)\right)$ by $(4.15)$, it follows from Propositions 2.3 and 4.1 that $v_{\nu}$ is bounded in $L^{\infty}\left(Q_{T}\right)$ and in $L^{\infty}\left(0, T, W^{1, p}(\Omega)\right) \forall p \in[2,+\infty)$. Therefore, we infer that for some sequence $\nu_{k} \searrow 0$ and some functions

$$
\begin{aligned}
v & \in L^{\infty}\left(0, T ; W^{1, p}(\Omega)\right) \quad \forall p \in[2,+\infty), \\
\omega & \in L^{\infty}\left(0, T ; L_{\theta}^{1}(\Omega) \cap L^{\infty}(\Omega)\right) \\
\text { and } \quad l & \in L^{\infty}(0, T),
\end{aligned}
$$

we have that

$$
\begin{aligned}
v_{\nu_{k}} \rightarrow v & \text { in } L^{\infty}\left(0, T ; W^{1, p}(\Omega)\right)-\text { weak } *, \forall p \in[2,+\infty) \\
\omega_{\nu_{k}} \rightarrow \omega & \text { in } L^{\infty}\left(Q_{T}\right)-\text { weak * } \\
l_{\nu_{k}} \rightarrow l & \text { in } L^{\infty}(0, T)-\text { weak * }
\end{aligned}
$$

as $k \rightarrow+\infty$.

Fix a number $q \in(1,2)$ and set $\Omega_{R}:=\{y \in \Omega ; 1<|y|<R\}$ for any $R>1$. Let

$$
V_{q, R}:=\left\{\varphi \in W_{0}^{1, q}\left(\Omega_{R}\right) ; \operatorname{div} \varphi=0\right\} .
$$

By the Sobolev embedding theorem $V_{q, R} \subset L^{2}\left(\Omega_{R}\right)$ for any $q \in(1,2)$. We claim that $\left(v_{\nu}\right)_{t}$ is bounded in $L^{2}\left(0, T,\left(V_{q, R}\right)^{\prime}\right)$. Indeed, taking the inner product of $(3.1)$ by $\varphi(t)$, where $\varphi \in L^{2}\left(0, T, V_{q, R}\right)$, and integrating by parts we obtain for a.e. $t \in(0, T)$

$$
\int_{\Omega_{R}}\left(v_{\nu}\right)_{t} \cdot \varphi \mathrm{d} y=-\nu \int_{\Omega_{R}} \nabla v_{\nu}: \nabla \varphi \mathrm{d} y+\int_{\Omega_{R}}\left(\left(l_{\nu}-v_{\nu}\right) \cdot \nabla v_{\nu}\right) \cdot \varphi \mathrm{d} y
$$


hence, by Hölder inequality,

$$
\left|\int_{\Omega_{R}}\left(v_{\nu}\right)_{t} \cdot \varphi \mathrm{d} y\right| \leq \nu\left\|\nabla v_{\nu}\right\|_{L^{q^{\prime}}\left(\Omega_{R}\right)} \cdot\|\nabla \varphi\|_{L^{q}\left(\Omega_{R}\right)}+\left(|l|+\left\|v_{\nu}\right\|_{L^{\infty}\left(\Omega_{R}\right)}\right)\left\|\nabla v_{\nu}\right\|_{L^{q^{\prime}}\left(\Omega_{R}\right)} \cdot\|\varphi\|_{L^{q}\left(\Omega_{R}\right)}
$$

where $q^{\prime}>2$ denotes the conjugate exponent of $q$. We conclude that $\left(v_{\nu}\right)_{t}$ is bounded in $L^{2}\left(0, T,\left(V_{q, R}\right)^{\prime}\right)$. Let $p>2$. Observing that the first embedding in

$$
W^{1, p}\left(\Omega_{R}\right) \subset C\left(\overline{\Omega_{R}}\right) \subset L^{2}\left(\Omega_{R}\right) \subset\left(V_{q, R}\right)^{\prime}
$$

is compact, we deduce from ([29], Cor. 4), that $\left(v_{\nu}\right)_{\nu>0}$ is relatively compact in $C\left(\overline{\Omega_{R}} \times[0, T]\right)$ for any $R>0$. Therefore, we obtain that

$$
v \in \mathcal{B}\left(\overline{Q_{T}}\right)
$$

and that $v_{\nu_{k}}$ converges to $v$ uniformly on each compact subset of $\bar{\Omega} \times[0, T]$ as $k \rightarrow+\infty$. On the other hand, using the identity

$$
v_{\nu_{k}} \cdot n=l_{\nu_{k}} \cdot n \quad \text { on } \partial B \times[0, T]
$$

valid for all $k \geqslant 0$, and the uniform convergence of $v_{\nu_{k}}$ to $v$ on $\partial B \times[0, T]$, we infer that $l_{\nu_{k}}$ converges uniformly to $l$ on $[0, T]$, hence $l \in C([0, T])$.

For any $\varphi \in L^{2}(0, T, \mathcal{V})$ we have by $(3.20)$ and $(3.29)$

$$
\int_{0}^{T}\left\{\left(v_{\nu_{k}}^{\prime}, \varphi\right)_{\rho}+\nu_{k} \int_{\Omega} \nabla v_{\nu_{k}}: \nabla \varphi \mathrm{d} y-\nu_{k} \int_{\partial B}\left(v_{\nu_{k}}-l_{\nu_{k}}\right) \cdot\left(\varphi-l_{\varphi}\right) \mathrm{d} \Gamma\right\} \mathrm{d} t=\int_{0}^{T} \int_{\Omega} f_{\nu_{k}} \cdot \varphi \mathrm{d} y \mathrm{~d} t
$$

where $f_{\nu_{k}}:=\left(l_{\nu_{k}}-v_{\nu_{k}}\right) \cdot \nabla v_{\nu_{k}}$. Noticing that $\mathcal{V}$ is dense in $\mathcal{H}$ and identifying $\mathcal{H}$ with $\mathcal{H}^{\prime}$, we obtain the diagram

$$
\mathcal{V} \subset \mathcal{H} \equiv \mathcal{H}^{\prime} \subset \mathcal{V}^{\prime}
$$

Therefore, we may write

$$
\left(v_{\nu_{k}}^{\prime}, \varphi\right)_{\rho}=\left\langle v_{\nu_{k}}^{\prime}, \varphi\right\rangle
$$

where the symbol $\langle\cdot, \cdot\rangle$ denotes the duality pairing between $\mathcal{V}^{\prime}$ and $\mathcal{V}$. As $\left(v_{\nu_{k}}\right)$ is bounded in $L^{2}\left(0, T, H^{1}(\Omega)\right) \cap$ $L^{\infty}\left(Q_{T}\right)$ and $\left(l_{\nu_{k}}\right)$ is bounded in $L^{\infty}(0, T)$, we infer from $(5.2)$ that $\left(v_{\nu_{k}}^{\prime}\right)$ is bounded in $L^{2}\left(0, T, \mathcal{V}^{\prime}\right)$. Extracting a subsequence if necessary, we may assume that $v_{\nu_{k}}^{\prime} \rightarrow v^{\prime}$ in $L^{2}\left(0, T, \mathcal{V}^{\prime}\right)$. As

$$
\left(l_{\nu_{k}}-v_{\nu_{k}}\right) \varphi \rightarrow(l-v) \varphi \quad \text { in } L^{2}\left(Q_{T}\right)
$$

taking the limit in $(5.2)$ we obtain

$$
\int_{0}^{T}\left\{\left\langle v^{\prime}, \varphi\right\rangle+\int_{\Omega}((v-l) \cdot \nabla v) \cdot \varphi \mathrm{d} y\right\} \mathrm{d} t=0 \quad \forall \varphi \in L^{2}(0, T, \mathcal{V})
$$

Obviously, (3.2), (3.3), (3.6) and (3.7) hold true. On the other hand $\left[\mathcal{V}, \mathcal{V}^{\prime}\right]_{\frac{1}{2}}=\mathcal{H}$, hence by a classical result in $[23]$

and we infer from Hölder inequality that

$$
v \in C([0, T], \mathcal{H})
$$

$$
v \in C\left([0, T], L^{p}(\Omega)\right) \quad \forall p \in[2,+\infty) .
$$

In particular, it follows from ([32], Lem. 1.4), that

$$
v \in C_{w}\left([0, T], W^{1, p}(\Omega)\right) \quad \forall p \in[2,+\infty)
$$


and that

$$
\lim _{|y| \rightarrow+\infty} v(y, t)=0 \quad \forall t \in[0, T] .
$$

We now turn to the equation satisfied by $\omega$. Using (4.2), (4.12) may be rewritten as

$$
\omega_{\nu_{k}}^{\prime}+\operatorname{div}\left(\left(v_{\nu_{k}}-l_{\nu_{k}}\right) \omega_{\nu_{k}}\right)-\nu_{k} \Delta \omega_{\nu_{k}}=0
$$

Clearly, $\left(v_{\nu_{k}}-l_{\nu_{k}}\right) \omega_{\nu_{k}} \rightarrow(v-l) \omega$ in $L^{\infty}\left(Q_{T}\right)$ - weak $*$, hence, letting $k \rightarrow+\infty$ in (5.5) we obtain

$$
\omega^{\prime}+\operatorname{div}((v-l) \omega)=0 \quad \text { in } \mathcal{D}^{\prime}\left(Q_{T}\right)
$$

Finally, passing to the limit in (4.11), (5.1) we get

$$
\begin{aligned}
\omega & =\operatorname{curl} v \\
\left|\int_{\partial B} v \cdot \tau \mathrm{d} \Gamma\right| & \leqslant\left\|\omega_{0}\right\|_{L^{1}(\Omega)}
\end{aligned}
$$

\subsection{Existence of a classical solution of (1.8)-(1.13)}

In this section we prove that all the equations in (1.8)-(1.13) are satisfied in the classical sense. More precisely, we prove that $v, \nabla v, v_{t}$ and $\nabla q$ belong to $\mathcal{B}\left(\overline{Q_{T}}\right)$. We begin with the following result.

Lemma 5.1. There exists a constant $H>0$ such that for all $y, z \in \bar{\Omega}$ and all $t \in[0, T]$

$$
|v(y, t)-v(z, t)| \leqslant H|y-z| \chi(|y-z|)
$$

where

$$
\chi(r):= \begin{cases}1 & \text { for } r \geqslant 1 \\ 1+\ln (1 / r) & \text { for } 0<r<1 .\end{cases}
$$

Proof. Applying Lemma 2.4 with $l=l(t)$, we may write

$$
v(y, t)=d_{1}(y, t)+v_{1}(y, t)
$$

where $d_{1} \in C\left([0, T], W^{1, \infty}(\Omega)\right)$ satisfies $d_{1}(y, t)=l(t)$ for $|y| \leqslant 2, d_{1}(y, t)=0$ for $|y| \geqslant 3$, and $v_{1}$ fulfils $\operatorname{curl} v_{1}=\omega_{1}:=\omega-\operatorname{curl} d_{1}$ and $\operatorname{div} v_{1}=0$ in $\Omega, v_{1} \cdot n=0$ on $\partial B, \lim _{|y| \rightarrow+\infty} v_{1}(y, t)=0$, and $\int_{\partial B} v_{1} \cdot \tau \mathrm{d} \Gamma=$ $\int_{\partial B} v \cdot \tau \mathrm{d} \Gamma:=C(t)$. (Note that the function $C(t)$ is continuous.) Then, by virtue of ([22], Lem. 2.14),

$$
v_{1}(y, t)=\operatorname{curl} G\left(\omega_{1}\right)(y, t)+\lambda_{1}(t) u_{1}(y)
$$

where $\lambda_{1}(t)=C(t)-\int_{\partial B} \operatorname{curl} G\left(\omega_{1}\right) \cdot \tau \mathrm{d} \Gamma$ and $u_{1} \in W^{1, \infty}(\Omega)$ is some irrotational and solenoidal flow satisfying $u_{1} \cdot n=0$ on $\partial B, \int_{\partial B} u_{1} \cdot \tau \mathrm{d} \Gamma=1$ and $u_{1}(y) \rightarrow 0$ as $|y| \rightarrow+\infty$. (See [22], Lem. 1.5, for the existence of the vector field $u_{1}$.) Then, by virtue of ([22], Lem. 2.4), $v_{1}$ and $v$ satisfy (5.9).

Remark 5.2. In [22] $\lambda_{1}$ takes the following form

$$
\lambda_{1}(t)=\int_{\partial B} a \cdot \tau \mathrm{d} \Gamma-\int_{\partial B} \operatorname{curl} G\left(\omega_{1}\right) \cdot \tau \mathrm{d} \Gamma
$$

Although it is expected that $C(t)=C(0)=\int_{\partial B} a \cdot \tau \mathrm{d} \Gamma$, this property has not yet been proved. The result in ([22], Lem. 2.4), remains nevertheless valid with this new definition of $\lambda_{1}(t)$. 
The vector field $v$ being quasi-Lipschitz (see (5.9)), it follows from Osgood's criterion (see e.g. [17], Cor. 6.2) that the Cauchy problem

$$
\left\{\begin{aligned}
\frac{\mathrm{d} y}{\mathrm{~d} t} & =v(y, t)-l(t) \\
y\left(t_{0}\right) & =y_{0}
\end{aligned}\right.
$$

has a unique solution $y(t)$. We may therefore define the flow associated to $v-l$ as the solution of the following system

$$
\left\{\begin{aligned}
\frac{\mathrm{d}}{\mathrm{d} s} U_{s, t}(y) & =v\left(U_{s, t}(y), s\right)-l(s), \\
U_{t, t}(y) & =y .
\end{aligned}\right.
$$

As $v \in \mathcal{B}\left(\overline{Q_{T}}\right), l \in C([0, T])$ and $(v-l) \cdot n=0$ on $\partial B \times[0, T]$, we see that $U_{s, t}(y)$ is defined for all $(s, t, y) \in$ $[0, T]^{2} \times \bar{\Omega}$. The following result comes from $[21]$.

Lemma 5.3. There exist two constants $\delta>0$ and $L>0$ such that

$$
\left|U_{s, t}(y)-U_{\bar{s}, \bar{t}}(\bar{y})\right| \leq L\left(|s-\bar{s}|^{\delta}+|t-\bar{t}|^{\delta}+|y-\bar{y}|^{\delta}\right) \quad \forall s, \bar{s}, t, \bar{t} \in[0, T], \forall y, \bar{y} \in \bar{\Omega}
$$

The following uniqueness result is similar to a result given in ([24], Proof of Thm. 2.5). Its proof is left to the reader.

Lemma 5.4. Let $v \in L^{\infty}\left(Q_{T}\right)$ be such that $\operatorname{div} v=0$ on $\Omega \times(0, T)$ and $v \cdot n=0$ on $\partial B \times(0, T)$, and let $\omega \in L^{\infty}\left(0, T, L^{1}(\Omega) \cap L^{\infty}(\Omega)\right)$ be a solution of

$$
\frac{\partial \omega}{\partial t}+\operatorname{div}(v \omega)=0 \quad \text { in } \mathcal{D}^{\prime}(\Omega \times(0, T))
$$

such that $\omega_{\mid t=0}=0$. Then $\omega \equiv 0$.

Let $\bar{\omega}(y, t):=\omega_{0}\left(U_{0, t}(y)\right)$, where $\omega_{0}:=\operatorname{curl} a$. As $\operatorname{div}(v-l)=0$, we infer as in $([22]$, pp. 70-71), that for all $t \in[0, T]$

$$
\int_{\Omega}|\bar{\omega}(y, t)| \mathrm{d} y=\int_{\Omega}\left|\omega_{0}(y)\right| \mathrm{d} y
$$

hence $\bar{\omega} \in L^{\infty}\left(0, T, L^{1}(\Omega) \cap L^{\infty}(\Omega)\right)$. It follows from Lemma 5.4 (applied to $\omega-\bar{\omega}$ and $v-l$ ) that

$$
\omega(y, t)=\bar{\omega}(y, t)=\omega_{0}\left(U_{0, t}(y)\right)
$$

Using once again the measure preserving property of $U_{s, t}(y)$, one may show that

$$
\int_{\Omega} \omega(y, t) \mathrm{d} y=\int_{\Omega} \omega_{0}(y) \mathrm{d} y
$$

hence

On the other hand, we infer from Lemma 5.3 that

$$
C(t)=\int_{\partial B} v \cdot \tau \mathrm{d} \Gamma=\text { Const. }
$$

$$
\omega \in C^{\gamma \lambda, 0}\left(\overline{Q_{T}}\right) \cap L^{\infty}\left(0, T, L_{\theta}^{1}(\Omega)\right) .
$$

Then we derive the following result.

Lemma 5.5. $\frac{\partial v}{\partial y_{j}} \in \mathcal{B}\left(\overline{Q_{T}}\right)$ for $j=1,2$. 
The proof is virtually the same as the one for ([22], Lem. 2.10). The following result contains the fact that $\nabla v \in C\left([0, T], L^{2}(\Omega)\right)$, which will be used later when proving that $v^{\prime}=\mathrm{d} v / \mathrm{d} t \in \mathcal{B}\left(\overline{Q_{T}}\right)$.

Lemma 5.6. $v \in C\left([0, T], L^{p}(\Omega)\right)$ for any $p \in(2,+\infty]$, and $\nabla v \in C\left([0, T], L^{p}(\Omega)\right)$ for any $p \in(1,+\infty)$.

Proof. We need the following

Claim. $\omega \in C\left([0, T], L^{1}(\Omega) \cap L^{\infty}(\Omega)\right)$.

We readily infer from $\omega \in L^{\infty}\left(0, T, L_{\theta}^{1}(\Omega)\right) \cap C\left(\overline{Q_{T}}\right)$ that $\omega \in C\left([0, T], L^{1}(\Omega)\right)$. On the other hand, $\omega_{0}$ is Hölder continuous on $\bar{\Omega}$ by assumption, and we infer from Lemma 5.3 that

$$
\left|U_{0, t}(y)-U_{0, t^{\prime}}(y)\right| \leqslant L\left|t-t^{\prime}\right|^{\delta} \quad \forall y \in \bar{\Omega}, \forall t, t^{\prime} \in[0, T] .
$$

Thus, the vorticity $\omega(y, t)=\omega_{0}\left(U_{0, t}(y)\right)$ belongs to the space $C\left([0, T], L^{\infty}(\Omega)\right)$. The claim is proved.

The proof of the lemma is completed by using Proposition 2.3, the claim, and the fact that $l \in C([0, T])$ and $\int_{\partial B} v \cdot \tau \mathrm{d} \Gamma=$ Const.

Lemma 5.7. $v^{\prime} \in \mathcal{B}\left(\overline{Q_{T}}\right) \cap C\left([0, T], L^{2}(\Omega)\right)$.

Proof. Let $\varphi \in L^{2}(0, T ; \mathcal{V})$. By $(5.3)$ we have that

$$
\int_{0}^{T}\left\{\left\langle v^{\prime}, \varphi\right\rangle+\int_{\Omega}((v-l) \cdot \nabla v) \cdot \varphi \mathrm{d} y\right\} \mathrm{d} t=0 .
$$

Let $f$ denote the function $((v-l) \cdot \nabla) v$ extended by 0 on $B$. We infer from Lemma 5.6 that $f \in C\left([0, T] ; L^{2}\left(\mathbb{R}^{2}\right)\right)$, hence $\mathbb{P} f \in C([0, T] ; \mathcal{H})$. It follows that

$$
\int_{\Omega}((v-l) \cdot \nabla v) \cdot \varphi \mathrm{d} y=(f, \varphi)_{\rho}=(\mathbb{P} f, \varphi)_{\rho}=\langle\mathbb{P} f, \varphi\rangle .
$$

Thus

which implies that

$$
\int_{0}^{T}\left\langle v^{\prime}+\mathbb{P} f, \varphi\right\rangle \mathrm{d} t=0 \quad \forall \varphi \in L^{2}(0, T ; \mathcal{V})
$$

$$
v^{\prime}+\mathbb{P} f=0 \quad \text { in } L^{2}\left(0, T ; \mathcal{V}^{\prime}\right)
$$

Thus $v^{\prime} \in C([0, T], \mathcal{H})$ and $l^{\prime}=l_{v^{\prime}} \in C([0, T])$. We now decompose $v$ as

$$
v(y, t)=v_{2}(y, t)+d_{1}(y, t)+C d_{2}(y)
$$

where $v_{2}$ solves $(2.19)-(2.23)$ (with $\left.\omega_{2}(y, t)=\omega(y, t)-\operatorname{curl} d_{1}(y, t)-C \operatorname{curl} d_{2}(y)\right), d_{1}\left(\operatorname{resp}\right.$. $\left.d_{2}\right)$ is given by Lemma 2.4 (resp. Lem. 2.5), and $C=\int_{\partial B} a \cdot \tau \mathrm{d} \Gamma$. Derivating with respect to time in (5.11), we obtain

$$
v^{\prime}=v_{2}^{\prime}+d_{1}^{\prime}
$$

As $l^{\prime} \in C([0, T]), d_{1}^{\prime} \in \mathcal{B}\left(\overline{Q_{T}}\right)$. The fact that $v_{2}^{\prime} \in \mathcal{B}\left(\overline{Q_{T}}\right)$ may be found in $([22]$, Proof of Lem. 2.11). Therefore, $v^{\prime} \in \mathcal{B}\left(\overline{Q_{T}}\right)$.

Corollary 5.8. $v \in C^{1}\left([0, T], L^{2}(\Omega)\right) \cap C\left([0, T], H^{1}(\Omega)\right)$.

Proof. This is a direct consequence of (5.4), and of Lemmas 5.6 and 5.7. 
It remains to prove the existence of a pressure $q(y, t)$ satisfying $(1.8),(1.11)$ in a classical sense. As $v^{\prime} \in$ $C([0, T], \mathcal{H})$, we infer from (5.3) that for every $t \in[0, T]$

$$
m l^{\prime} \cdot l_{\phi}+\int_{\Omega}\left(v^{\prime}+(v-l) \cdot \nabla v\right) \cdot \phi \mathrm{d} y=0 \quad \forall \phi \in \mathcal{V} .
$$

In particular,

$$
\int_{\Omega}\left(v^{\prime}+(v-l) \cdot \nabla v\right) \cdot \phi \mathrm{d} y=0 \quad \forall \phi \in C_{0}^{\infty}(\Omega) \text { with } \operatorname{div} \phi=0 .
$$

By ([32], Props. 1.1 and 1.2), there exists a function $q \in L^{2}\left(0, T, \hat{H}^{1}(\Omega)\right)$ such that for a.e. $t \in(0, T)$

$$
v^{\prime}+(v-l) \cdot \nabla v+\nabla q=0 .
$$

As $v^{\prime}$ and $(v-l) \cdot \nabla v$ belong to $\mathcal{B}\left(\overline{Q_{T}}\right) \cap C\left([0, T], L^{2}(\Omega)\right)$, adding a function of time to $q$ if necessary we see that $q \in C\left([0, T], C^{1}(\bar{\Omega})\right) \cap C\left([0, T], \hat{H}^{1}(\Omega)\right)$. Picking any $\phi \in \mathcal{V}$, we infer from (5.12)-(5.13) and the divergence formula that

$$
m l^{\prime} \cdot l_{\phi}=\int_{\Omega} \nabla q \cdot \phi \mathrm{d} y=\int_{\Omega} \operatorname{div}(q \phi) \mathrm{d} y=\int_{\partial B} q n \cdot \phi \mathrm{d} \Gamma .
$$

Therefore (1.11) holds true.

\subsection{Uniqueness of the solution}

Now, we investigate the uniqueness of the solutions of the problem (1.1)-(1.6). Recall that by using the change of variables (1.7), we obtain the equivalent model (1.8)-(1.13).

Assume given two classical solutions $\left(v^{1}, q^{1}, l^{1}\right)$ and $\left(v^{2}, q^{2}, l^{2}\right)$ of $(1.8)-(1.13)$. We then introduce the functions

which fulfil the following system

$$
v=v^{1}-v^{2} \quad q=q^{1}-q^{2} \quad l=l^{1}-l^{2},
$$

$$
\begin{aligned}
\frac{\partial v}{\partial t}+\left(\left(v^{1}-l^{1}\right) \cdot \nabla\right) v+((v-l) \cdot \nabla) v^{2}+\nabla q=0, & \text { in } \Omega \times[0, T], \\
\operatorname{div} v=0, & \text { in } \Omega \times[0, T], \\
v \cdot n=l \cdot n, & \text { on } \partial B \times[0, T], \\
m l^{\prime}=\int_{\partial B} q n \mathrm{~d} \Gamma, & \text { in }[0, T], \\
v(y, 0)=0, & \forall y \in \Omega, \\
l(0)=0 . &
\end{aligned}
$$

In order to prove that $(v, q, l)=(0,0,0)$, we consider some energy estimate for $(5.15)-(5.20)$.

Firstly, by multiplying (5.15) by $v$ and by integrating over $\Omega \times(0, t)$, we have that

$$
\begin{aligned}
0=\int_{0}^{t} \int_{\Omega} v_{t} \cdot v \mathrm{~d} y \mathrm{~d} s+\int_{0}^{t} \int_{\Omega}\left(\left(v^{1}-l^{1}\right)\right. & \cdot \nabla) v \cdot v \mathrm{~d} y \mathrm{~d} s \\
& +\int_{0}^{t} \int_{\Omega}((v-l) \cdot \nabla) v^{2} \cdot v \mathrm{~d} y \mathrm{~d} s+\int_{0}^{t} \int_{\Omega} \nabla q \cdot v \mathrm{~d} y \mathrm{~d} s=I_{1}+I_{2}+I_{3}+I_{4} .
\end{aligned}
$$

We now study each integral term. We easily have that

$$
I_{1}=\frac{1}{2} \int_{\Omega}|v(t)|^{2} \mathrm{~d} y .
$$


Next, some integrations by part give that

$$
I_{2}=0
$$

On the other hand, we have that

$$
\begin{aligned}
I_{3} & =\int_{0}^{t} \int_{\Omega}((v-l) \cdot \nabla) v^{2} \cdot v \mathrm{~d} y \mathrm{~d} s \\
& =\int_{0}^{t} \int_{\Omega}(v \cdot \nabla) v^{2} \cdot v \mathrm{~d} y \mathrm{~d} s-\int_{0}^{t} \int_{\Omega}(l \cdot \nabla) v^{2} \cdot v \mathrm{~d} y \mathrm{~d} s \\
& =I_{31}+I_{32} .
\end{aligned}
$$

We can estimate each part:

and

$$
\left|I_{31}\right| \leqslant\left\|\nabla v^{2}\right\|_{L^{\infty}\left(Q_{T}\right)} \int_{0}^{t} \int_{\Omega}|v|^{2} \mathrm{~d} y \mathrm{~d} s
$$

$$
\begin{aligned}
\left|I_{32}\right| & \leqslant \int_{0}^{t}|l(s)|\left(\int_{\Omega}\left|\nabla v^{2}\right|^{2} \mathrm{~d} y\right)^{1 / 2}\left(\int_{\Omega}|v|^{2} \mathrm{~d} y\right)^{1 / 2} \mathrm{~d} s \\
& \leqslant \frac{1}{2}\left\|v^{2}\right\|_{L^{\infty}\left(0, T, H^{1}(\Omega)\right)}\left[\int_{0}^{t}\left(\int_{\Omega}|v|^{2} \mathrm{~d} y+|l|^{2}\right) \mathrm{d} s\right] .
\end{aligned}
$$

Finally we have that

Thus, we have that

$$
I_{4}=\frac{m}{2} \int_{0}^{t} \frac{\mathrm{d}}{\mathrm{d} s}\left(|l|^{2}\right) \mathrm{d} s=\frac{m}{2}|l(t)|^{2}
$$

$$
\int_{\Omega}|v(t)|^{2}+m|l(t)|^{2} \leqslant C\left[\int_{0}^{t}\left(\int_{\Omega}|v|^{2} \mathrm{~d} y+m|l|^{2}\right) \mathrm{d} s\right]
$$

and by Gronwall's Lemma, we obtain that

$$
v=0 \quad \text { in } \quad \Omega \times(0, T) \text { and } \quad l=0 \quad \text { in }(0, T) .
$$

Using (5.15) we conclude that $\nabla q=0$ in $\Omega \times(0, T)$, which proves that the solution of our problem is unique (up to an arbitrary function of $t$ for $q$ ). The proof of Theorem 1.1 is achieved.

Acknowledgements. The authors gratefully acknowledge several discussions with Carlos Conca, Chérif Amrouche and Saïd Benachour. The first author was partially supported by FONDECYT grant 1030943 and INRIA-CONICYT and the second and the third authors were partially supported by INRIA-CONICYT and by the project CORIDA (INRIA).

\section{REFERENCES}

[1] H. Brezis, Analyse fonctionnelle. Collection Mathématiques Appliquées pour la Maîtrise. [Collection of Applied Mathematics for the Master's Degree]. Masson, Paris (1983). Théorie et Applications. [Theory and applications].

[2] C. Conca, J. San Martín H. and M. Tucsnak, Existence of solutions for the equations modelling the motion of a rigid body in a viscous fluid. Comm. Partial Differential Equations 25 (2000) 1019-1042.

[3] J.-M. Coron, On the controllability of 2-D incompressible perfect fluids. J. Math. Pures Appl. (9) 75 (1996) $155-188$.

[4] J.-M. Coron, On the null asymptotic stabilization of the two-dimensional incompressible Euler equations in a simply connected domain. SIAM J. Control Optim. 37 (1999) 1874-1896 (electronic).

[5] B. Desjardins and M.J. Esteban, Existence of weak solutions for the motion of rigid bodies in a viscous fluid. Arch. Ration. Mech. Anal. 146 (1999) 59-71. 
[6] B. Desjardins and M.J. Esteban, On weak solutions for fluid-rigid structure interaction: compressible and incompressible models. Comm. Partial Differential Equations 25 (2000) 1399-1413.

[7] E. Feireisl, On the motion of rigid bodies in a viscous fluid. Appl. Math. 47 (2002) 463-484. Mathematical theory in fluid mechanics, Paseky (2001).

[8] E. Feireisl, On the motion of rigid bodies in a viscous compressible fluid. Arch. Ration. Mech. Anal. 167 (2003) $281-308$.

[9] E. Feireisl, On the motion of rigid bodies in a viscous incompressible fluid. J. Evol. Equ. 3 (2003) 419-441. Dedicated to Philippe Bénilan.

[10] G.P. Galdi, On the steady self-propelled motion of a body in a viscous incompressible fluid. Arch. Ration. Mech. Anal. 148 (1999) 53-88.

[11] G.P. Galdi and A.L. Silvestre, Strong solutions to the problem of motion of a rigid body in a Navier-Stokes liquid under the action of prescribed forces and torques. In Nonlinear problems in mathematical physics and related topics, I. Int. Math. Ser. (N.Y.), Kluwer/Plenum, New York 1 (2002) 121-144.

[12] T. Gallay and C.E. Wayne, Invariant manifolds and the long-time asymptotics of the Navier-Stokes and vorticity equations on $\mathbf{R}^{2}$. Arch. Ration. Mech. Anal. 163 (2002) 209-258.

[13] N.S. Gilbarg and D. Trudinger, Elliptic partial differential equations of second order. Classics in Mathematics. Springer-Verlag, Berlin (2001). Reprint of the 1998 edition.

[14] O. Glass, Exact boundary controllability of 3-D Euler equation. ESAIM: COCV 5 (2000) 1-44 (electronic).

[15] C. Grandmont and Y. Maday, Existence for an unsteady fluid-structure interaction problem. ESAIM: M2AN 34 (2000) 609636.

[16] M.D. Gunzburger, H.-C. Lee and G.A. Seregin, Global existence of weak solutions for viscous incompressible flows around a moving rigid body in three dimensions. J. Math. Fluid Mech. 2 (2000) 219-266.

[17] P. Hartman, Ordinary differential equations. Birkhäuser Boston, MA, second edition (1982).

[18] K.-H. Hoffmann and V.N. Starovoitov, On a motion of a solid body in a viscous fluid. Two-dimensional case. Adv. Math. Sci. Appl. 9 (1999) 633-648.

[19] K.-H. Hoffmann and V.N. Starovoitov, Zur Bewegung einer Kugel in einer zähen Flüssigkeit. Doc. Math. 5 (2000) 15-21 (electronic).

[20] N.V. Judakov, The solvability of the problem of the motion of a rigid body in a viscous incompressible fluid. Dinamika Splošn. Sredy, (Vyp. 18 Dinamika Zidkost. so Svobod. Granicami) 255 (1974) 249-253.

[21] T. Kato, On classical solutions of the two-dimensional nonstationary Euler equation. Arch. Rational Mech. Anal. 25 (1967) $188-200$.

[22] K. Kikuchi, Exterior problem for the two-dimensional Euler equation. J. Fac. Sci. Univ. Tokyo Sect. IA Math. 30 (1983) 63-92.

[23] J.-L. Lions and E. Magenes, Non-homogeneous boundary value problems and applications. Vol. I. Springer-Verlag, New York (1972). Translated from the French by P. Kenneth, Die Grundlehren der mathematischen Wissenschaften, Band 181.

[24] P.-L. Lions, Mathematical topics in fluid mechanics. Vol. 1, The Clarendon Press Oxford University Press, New York. Incompressible models, Oxford Science Publications. Oxford Lect. Ser. Math. Appl. 3 (1996).

[25] C. Rosier and L. Rosier, Well-posedness of a degenerate parabolic equation issuing from two-dimensional perfect fluid dynamics. Appl. Anal. 75 (2000) 441-465.

[26] J. San Martín H., V. Starovoitov and M. Tucsnak, Global weak solutions for the two dimensional motion of several rigid bodies in an incompressible viscous fluid. Arch. Rational Mech. Anal. 161 (2002) 113-147.

[27] D. Serre, Chute libre d'un solide dans un fluide visqueux incompressible. Existence. Japan J. Appl. Math. 4 (1987) 99-110.

[28] A.L. Silvestre, On the self-propelled motion of a rigid body in a viscous liquid and on the attainability of steady symmetric self-propelled motions. J. Math. Fluid Mech. 4 (2002) 285-326.

[29] J. Simon, Compact sets in the space $L^{p}(0, T ; B)$. Ann. Mat. Pura Appl. (4) 146 (1987) 65-96.

[30] T. Takahashi, Analysis of strong solutions for the equations modeling the motion of a rigid-fluid system in a bounded domain. Adv. Differential Equations 8 (2003) 1499-1532.

[31] T. Takahashi and M. Tucsnak, Global strong solutions for the two-dimensional motion of an infinite cylinder in a viscous fluid. J. Math. Fluid Mech. 6 (2004) 53-77.

[32] R. Temam, Navier-Stokes equations. North-Holland Publishing Co., Amsterdam, third edition (1984). Theory and numerical analysis, with an appendix by F. Thomasset.

[33] J.L. Vázquez and E. Zuazua, Large time behavior for a simplified 1D model of fluid-solid interaction. Comm. Partial Differential Equations 28 (2003) 1705-1738. 